\title{
The gravity wave momentum flux in hydrostatic flow with directional shear over elliptical mountains
}

Article

Accepted Version

Teixeira, M. A. C. and Yu, C. L. (2014) The gravity wave momentum flux in hydrostatic flow with directional shear over elliptical mountains. European Journal of Mechanics \& Fluids B: Fluids, 47. pp. 16-31. ISSN 0997-7546 doi: https://doi.org/10.1016/j.euromechflu.2014.02.004 Available at https://centaur.reading.ac.uk/36164/

It is advisable to refer to the publisher's version if you intend to cite from the work. See Guidance on citing.

Published version at: http://dx.doi.org/10.1016/j.euromechflu.2014.02.004

To link to this article DOI: http://dx.doi.org/10.1016/j.euromechflu.2014.02.004

Publisher: Elsevier

All outputs in CentAUR are protected by Intellectual Property Rights law, including copyright law. Copyright and IPR is retained by the creators or other copyright holders. Terms and conditions for use of this material are defined in the End User Agreement.

www.reading.ac.uk/centaur 
Central Archive at the University of Reading

Reading's research outputs online 


\title{
The gravity wave momentum flux in hydrostatic flow with directional shear over elliptical mountains
}

\author{
Miguel A. C. Teixeira*, Chau Lam Yu \\ Department of Meteorology, University of Reading, \\ Earley Gate, PO Box 243, Reading RG6 6BB, UK
}

\begin{abstract}
Semi-analytical expressions for the momentum flux associated with orographic internal gravity waves, and closed analytical expressions for its divergence, are derived for inviscid, stationary, hydrostatic, directionally-sheared flow over mountains with an elliptical horizontal cross-section. These calculations, obtained using linear theory conjugated with a third-order WKB approximation, are valid for relatively slowly-varying, but otherwise generic wind profiles, and given in a form that is straightforward to implement in drag parametrization schemes. When normalized by the surface drag in the absence of shear, a quantity that is calculated routinely in existing drag parametrizations, the momentum flux becomes independent of the detailed shape of the orography. Unlike linear theory in the $R i \rightarrow \infty$ limit, the present calculations account for shear-induced amplification or reduction of the surface drag, and partial absorption of the wave momentum flux at critical levels. Profiles of the normalized momentum fluxes obtained using this model and a linear numerical model without the WKB approximation are evaluated and
\end{abstract}

\footnotetext{
${ }^{*}$ Corresponding author. Tel.: +44 118 3785573; Fax: +44 1183788905 .

URL: M.A.Teixeira@reading.ac.uk (Miguel A. C. Teixeira)
} 
compared for two idealized wind profiles with directional shear, for different Richardson numbers $(R i)$. Agreement is found to be excellent for the first wind profile (where one of the wind components varies linearly) down to $R i=0.5$, while not so satisfactory, but still showing a large improvement relative to the $R i \rightarrow \infty$ limit, for the second wind profile (where the wind turns with height at a constant rate keeping a constant magnitude). These results are complementary, in the $R i \gtrsim O(1)$ parameter range, to Broad's generalization of the Eliassen-Palm theorem to 3D flow. They should contribute to improve drag parametrizations used in global weather and climate prediction models.

Keywords: Flow over orography, Gravity wave drag, Wave momentum flux, Directional wind shear, Linear theory, WKB approximation

\section{Introduction}

One of the many physical processes that are currently still unresolved in large-scale weather and climate prediction models is the effect of atmospheric gravity waves. These waves propagate in stratified fluids (such as the atmo-

sphere typically is) [1], and are predominantly forced by flow over orography or convection occurring at horizontal scales $(1 \mathrm{~km}-10 \mathrm{~km})$ smaller than the grid spacings used operationally.

Waves generated by flow over mountains, which constitute a sizable fraction of the total gravity waves, are known as mountain waves. These waves produce a surface drag on orography [2], whose reaction force decelerates the airflow, and must be parametrized to avoid substantial biases in the simulated global atmospheric circulation [3]. However, most well-known drag 
parametrizations are now outdated, having been developed in the 1990s [4, 5]. The part of these parametrizations that accounts for the impact of wave propagation on the surface drag is based on linear wave theory, neglecting a number of important physical processes, such as non-hydrostatic effects, variations of the wind and static stability with height, and obviously wave nonlinearity, to mention just a few.

Linear theory is useful for developing drag parametrizations because it allows the drag to be expressed as a function of key orographic and incoming flow parameters. While a treatment of nonlinearity is, by definition, beyond its capabilities, wind profile effects can, in principle, be incorporated, although for generic wind profiles no analytical solutions exist, which precludes the derivation of simple drag expressions. Nevertheless, vertical wind shear has decisive implications for drag parametrization, since it may cause divergence of the wave momentum flux, which corresponds to a non-zero value of the reaction force exerted by the orography on the atmosphere $[6,7]$.

Eliassen and Palm [8] demonstrated that the wave momentum flux in 2D flows is constant with height, even when the wind and static stability vary, except at levels where the wind vanishes (critical levels). This means that all the drag is exerted on the atmosphere at those particular discrete levels. More recently, however, Broad [9] showed that, in flows with directional shear over 3D mountains, critical levels (where the wind velocity is perpendicular to the horizontal wavenumber vector of a given spectral component of the waves) have a continuous distribution with height, and the variation of the wave momentum flux is coupled with the turning of the wind with height. Specifically, at a given level, the vertical derivative of the wave momentum 
flux vector (the momentum flux divergence) must be perpendicular to the wind velocity. This law, which is a generalization of the Eliassen-Palm theorem to $3 \mathrm{D}$, places a strong constraint on the force exerted by mountains on the atmosphere.

However, the exact dependence of the drag on key flow parameters can only be determined by solving the corresponding mountain wave problem. Since this is not feasible analytically in the case of generic wind and static stability profiles, numerical or approximate methods must be employed. In the second category, one possibility is to split the atmosphere into a number of layers within which the wind velocity and static stability have a simple form (e.g., $[10,11,12,13]$ ), and then obtain the complete wave solutions and the corresponding drag. However, this approach lacks generality, and its results are often too cumbersome to implement in parametrizations. An alternative approach is to assume that the wind profile varies relatively slowly with height, and adopt a WKB approximation to obtain the wave solutions. Despite its limitations, this approach is considerably more general, being valid for generic (albeit slowly varying) wind profiles, and therefore providing a leading-order correction to the drag due to variation of the wind with height.

Teixeira et al. [14] calculated the surface drag using linear theory with a second-order WKB approximation for sheared, stationary, hydrostatic flow over an axisymmetric mountain and Teixeira and Miranda [15] did the same for 2D mountains. This model was extended to mountains with an elliptical horizontal cross-section by Teixeira and Miranda [16]. Subsequently, the wave momentum flux was calculated, using the same kind of approach, 
for flows with directional shear over an axisymmetric mountain [17]. This elucidated the filtering effect of critical levels with a continuous distribution with height in such flows, where the wave momentum flux may not be totally absorbed at relatively low Richardson numbers $(R i)$, but rather filtered. Teixeira and Miranda [17] did not calculate the wave momentum flux for flows with unidirectional shear or over 2D mountains, where critical levels occur at discrete heights (as mentioned above). These very particular cases had been addressed previously for simple wind profiles (without invoking the WKB approximation) by Booker and Bretherton [18] and Grubišić and Smolarkiewicz [19].

In the most important weather prediction and climate models, such as that running at the European Centre for Medium-Range Weather Forecasts (ECMWF), the Earth's orography is approximated in each model grid box as a mountain with an elliptical horizontal cross-section, with height, width and orientation calculated statistically from the real orography [20,4]. This approach appeals to a superposition principle (whereby the waves in each grid box do not interact with those in adjacent ones) whose strict validity is not straightforward, even for linearized flow. However, the fact that the width of these elliptical mountains is likely to be substantially smaller than the grid box (because they represent unresolved orography), and the assumption that the flow is hydrostatic (thus having very limited lateral wave propagation, especially at the surface), are consistent with the adopted, single-column approach.

In the present study we will develop and test the theory for calculating wave momentum fluxes in linearized, hydrostatic, non-rotating flow with di- 
rectional shear over elliptical mountains. This theory provides nearly readyto-use momentum flux and momentum flux divergence expressions, which may easily be incorporated into drag parametrizations. The results will be compared to those produced by a linear numerical model that does not assume the WKB approximation. Nonlinear effects were addressed previously in some detail for an axisymmetric mountain via comparisons with numerical simulations [17], and should not differ too much qualitatively for an elliptical geometry. Since it seems hopeless at present to formulate a physically self-consistent nonlinear mountain wave theory that is simple enough to implement in drag parametrizations, we necglect nonlinear effects altogether and focus here instead on evaluating the accuracy of the WKB approximation.

The remainder of this paper is organized as follows. Section 2 presents the linear mountain wave theory using the WKB approximation on which the subsequent momentum flux calculations are based. In section 3, a linear numerical model that allows the treatment of arbitrary wind profiles (i.e. not assuming the WKB approximation) is briefly described. Section 4 explores the behaviour of the wave momentum flux with height for two representative idealized wind profiles. Finally, section 5 summarizes the main conclusions of this study.

\section{Linear WKB theory}

The vertical flux of horizontal momentum associated with mountain waves forced by an arbitrarily-shaped isolated obstacle is defined here as

$$
\left(M_{x}, M_{y}\right)=-\rho \int_{-\infty}^{+\infty} \int_{-\infty}^{+\infty}(u, v) w d x d y
$$


where $\rho$ is the density, $(u, v, w)$ is the velocity perturbation created by the waves, and $x$ and $y$ are the horizontal spatial coordinates. As in Teixeira and Miranda [17], this definition includes the minus sign, because the momentum flux is generally downward, and that convention makes it positive for a mean flow that is positive in the $x$ and/or $y$ direction.

Departing from linear theory with the Boussinesq approximation, assuming inviscid, non-rotating, stationary, hydrostatic flow and using additionally a WKB approximation to solve the Taylor-Goldstein equation (where the vertical wavenumber of the waves is expanded in a power series of a small parameter $\varepsilon$ proportional to $R i^{-1 / 2}$ [14] up to third order), it can be shown that the momentum flux, correct up to second-order in $\varepsilon$, is given by

$$
\begin{aligned}
& \left(M_{x}, M_{y}\right)(z)=4 \pi^{2} \rho_{0} N \int_{-\infty}^{+\infty} \int_{-\infty}^{+\infty} \frac{(k, l)|\hat{h}|^{2}}{\left(k^{2}+l^{2}\right)^{1 / 2}}\left|U_{0} k+V_{0} l\right| \operatorname{sgn}(U k+V l) \\
& \times[1-S(k, l, z)] e^{[S(k, l, z)-S(k, l, z=0)]} e^{-2 \pi H\left(z-z_{c}\right) C(k, l)} d k d l
\end{aligned}
$$

(see Equations (27)-(29) of [17]), where

$$
\begin{aligned}
S(k, l, z) & =\frac{1}{8} \frac{\left(U^{\prime} k+V^{\prime} l\right)^{2}}{N^{2}\left(k^{2}+l^{2}\right)}+\frac{1}{4} \frac{(U k+V l)\left(U^{\prime \prime} k+V^{\prime \prime} l\right)}{N^{2}\left(k^{2}+l^{2}\right)}, \\
C(k, l) & =\frac{N\left(k^{2}+l^{2}\right)^{1 / 2}}{\left|U_{c}^{\prime} k+V_{c}^{\prime} l\right|}\left[1-\frac{1}{8} \frac{\left(U_{c}^{\prime} k+V_{c}^{\prime} l\right)^{2}}{N^{2}\left(k^{2}+l^{2}\right)}\right] .
\end{aligned}
$$

In the above equations $\rho_{0}$ is a reference density (assumed to be constant), $z$ is height, $N$ and $(U, V)$ are the Brunt-Väisälä frequency and velocity of the mean incoming flow, $(k, l)$ is the horizontal wavenumber vector of the waves, and $\hat{h}(k, l)$ is the Fourier transform of the terrain elevation $h(x, y) . z_{c}$ is the height of the critical level, the subscript 0 denotes values taken at the surface $z=0$ and the subscript $c$ denotes values taken at the critical level, $H$ is the 
Heaviside step function and the primes denote differentiation with respect to $z$.

$N$ is taken here as constant, for simplicity, but that assumption may be relaxed. Is is reasonable to assume stationary flow, since the time scale for the onset of steady mountain waves is typically shorter than that over which the mean incoming flow evolves. The assumption of non-rotating flow is valid for the wave perturbations as long as $f a / U \ll 1$, where $f$ is the Coriolis parameter and $a$ is a measure of mountain width. Additionally, the hydrostatic assumption requires $N a / U \gg 1$. For the typical values in the atmosphere $N \approx 10^{-2} \mathrm{~s}^{-1}, U \approx 10 \mathrm{~ms}^{-1}$ and $f \approx 10^{-4} \mathrm{~s}^{-1}$, these two assumptions are simultaneously valid for $a \approx 10 \mathrm{~km}$. This is highly relevant for parametrization purposes, because it is known that the maximum contribution to mountain wave drag occurs under hydrostatic and non-rotating conditions [21]. Linearization, which is perhaps the most unrealistic, but crucial, assumption, is formally valid for $N h_{0} / U \ll 1$, where $h_{0}$ is a measure of mountain height. Using the same values of $N$ and $U$ as previously, this would imply $h_{0} \ll 1 \mathrm{~km}$. Errors produced by these approximations due to flow unsteadiness, non-hydrostatic effects or nonlinearity can be estimated using numerical simulations [17], but such simulations have proved unable to provide a tractable, self-consistent framework for parametrization.

Equation (2) satisfies up to third-order in the small perturbation parameter $\varepsilon$ the extension to 3D of Eliassen-Palm's theorem formulated by Broad $[9]$

$$
U(z) \frac{d M_{x}}{d z}+V(z) \frac{d M_{y}}{d z}=0,
$$

because any wavenumber that contributes to $d\left(M_{x}, M_{y}\right) / d z \neq 0$ at or below 
that order must satisfy $U k+V l=0$ (the critical level condition). As noted by Teixeira and Miranda [17], $S(k, l, z)$ encapsulates corrections to the momentum flux due to wind profile shear and curvature, while $C(k, l)$ contains the effects of critical levels, which may either totally absorb or simply filter the momentum flux, depending on the shear intensity.

\subsection{Elliptical geometry}

We intend to address flow over mountains with an elliptical horizontal cross-section. Without loss of generality, the coordinate system will be aligned with the major and minor axes of the ellipse, so that $a$ is the halfwidth of the mountain along $x$ and $b$ is the half-width along $y$. As in [16], it then becomes convenient to introduce a polar elliptical coordinate system in the wavenumber domain, in terms of which

$$
k=\frac{\kappa}{a} \cos \theta, \quad l=\frac{\kappa}{b} \sin \theta,
$$

where $\kappa$ is the radial coordinate and $\theta$ the azimuthal angle. Note that, unlike what is usual in polar coordinates $\kappa$ is dimensionless and does not express the magnitude of the horizontal wavenumber vector. Similarly, $\theta$ is not the angle this vector makes with the $x$ axis, although it can be related to it.

Using these coordinates, (2) can be rewritten as

$$
\begin{aligned}
& \left(M_{x}, M_{y}\right)=4 \pi^{2} \rho_{0} N b h_{0}^{2} \int_{0}^{+\infty} \kappa^{2}\left|\hat{h}^{\prime}(\kappa)\right|^{2} d \kappa \int_{0}^{2 \pi} \frac{(\cos \theta, \gamma \sin \theta)}{\left(\cos ^{2} \theta+\gamma^{2} \sin ^{2} \theta\right)^{1 / 2}} \\
& \times\left|U_{0} \cos \theta+\gamma V_{0} \sin \theta\right| \operatorname{sgn}(U \cos \theta+\gamma V \sin \theta)[1-S(\theta, z)] \\
& \times e^{[S(\theta, z)-S(\theta, z=0)]} e^{-2 \pi H\left(z-z_{c}\right) C(\theta)} d \theta,
\end{aligned}
$$

where

$$
S(\theta, z)=\frac{1}{8} \frac{\left(U^{\prime} \cos \theta+\gamma V^{\prime} \sin \theta\right)^{2}}{N^{2}\left(\cos ^{2} \theta+\gamma^{2} \sin ^{2} \theta\right)}
$$




$$
\begin{gathered}
+\frac{1}{4} \frac{(U \cos \theta+\gamma V \sin \theta)\left(U^{\prime \prime} \cos \theta+\gamma V^{\prime \prime} \sin \theta\right)}{N^{2}\left(\cos ^{2} \theta+\gamma \sin ^{2} \theta\right)} \\
C(\theta)=\frac{N\left(\cos ^{2} \theta+\gamma^{2} \sin ^{2} \theta\right)^{1 / 2}}{\left|U_{c}^{\prime} \cos \theta+\gamma V_{c}^{\prime} \sin \theta\right|}\left[1-\frac{1}{8} \frac{\left(U_{c}^{\prime} \cos \theta+\gamma V_{c}^{\prime} \sin \theta\right)^{2}}{N^{2}\left(\cos ^{2} \theta+\gamma^{2} \sin ^{2} \theta\right)}\right] .
\end{gathered}
$$

In these equations, $h_{0}$ is the height of the mountain, $\gamma=a / b$ is its horizontal aspect ratio, and $\hat{h}^{\prime}=\hat{h} /\left(h_{0} a b\right)$ is the dimensionless Fourier transform of the terrain elevation. The fact that the surface elevation has an elliptical horizontal cross-section has been used to simplify (7), as this means that $h(x, y)=h\left[(x / a)^{2}+(y / b)^{2}\right]$, and then (6) and the definition of Fourier transform imply that $\hat{h}(k, l)=\hat{h}(\kappa)$, as assumed in (7) (cf. [16]).

Equation (7) already shows that, as in the case of an axisymmetric mountain (cf. [17]) the momentum flux integral can be expressed as the product of an integral along the radial direction $\kappa$ and an integral along the azimuthal angle $\theta$. This turns out to be the key aspect that renders corrections to the momentum flux due to shear independent of the detailed shape of the orography, since when the momentum flux is normalized, for example, by the surface drag, the integral in the radial direction (which contains information about the shape of the orography along this direction), cancels out, and only the integral in $\theta$ remains. This simplification is only possible because of the hydrostatic assumption (see original discussion in [14]). It should also be noted that the functions $S(\theta, z)$ and $C(\theta)$ are also independent of $\kappa$ (and hence have been isolated in the integral over $\theta$ ).

\subsection{Momentum flux normalization}

The studies of Teixeira et al. [14] and Teixeira and Miranda [16, 17] highlighted difficulties in conveniently normalizing both components of the drag or of the momentum flux so that they take a value of 1 for high $R i$. 
One option when the drag was oblique to the axes of the adopted coordinate system was to normalize each drag component including the effect of shear by the corresponding component in the absence of shear. However, this strategy fails if one of the drag components in the absence of shear is zero. A better choice, which arises naturally from the calculations, is to express the momentum flux as a function of the two drag components without shear, since this corresponds to vector a transformation which may include rotation. This choice was adopted by Miranda et al. [22] for the surface drag for practical reasons, and the same will be done here next for the momentum flux. A different choice may, of course, be adopted strictly for the purpose of graphically representing these quantities.

The mean incoming flow velocity is expressed in elliptical polar coordinates as

$$
U=a \mu \cos \psi, \quad V=b \mu \sin \psi
$$

where $\mu$ is a radial measure of the wind intensity, but is not directly proportional to the wind speed, nor has dimensions of velocity, while $\psi$ is an azimuthal angle, which, however, does not coincide with the angle made by the wind with the $x$ axis. The advantage of this transformation is that, when (6) is also employed,

$$
U k+V l=\mu \kappa(\cos \theta \cos \psi+\sin \theta \sin \psi)=\mu \kappa \cos (\theta-\psi),
$$

and so critical levels, where $U k+V l=0$, can simply be expressed as levels where $\theta=\psi \pm \pi / 2$ (as in [17]). It should be stressed again, however, that neither $\psi$ nor $\theta$ have the simple interpretation that the equivalent quantities have for an axisymmetric mountain (where $\gamma=1$ ) when usual polar coordinates are used. 
If (10) is used in (7), the latter equation can be rewritten

$$
\begin{aligned}
& \left(M_{x}, M_{y}\right)=4 \pi^{2} \rho_{0} N a b \mu_{0} h_{0}^{2} \int_{0}^{+\infty} \kappa^{2}\left|\hat{h}^{\prime}(\kappa)\right|^{2} d \kappa \int_{0}^{2 \pi} \frac{(\cos \theta, \gamma \sin \theta)}{\left(\cos ^{2} \theta+\gamma^{2} \sin ^{2} \theta\right)^{1 / 2}} \\
& \times\left|\cos \left(\theta-\psi_{0}\right)\right| \operatorname{sgn}[\cos (\theta-\psi)][1-S(\theta, z)] e^{[S(\theta, z)-S(\theta, z=0)]} \\
& \times e^{-2 \pi H\left(z-z_{c}\right) C(\theta)} d \theta
\end{aligned}
$$

where $\psi_{0}=\psi(z=0)$ and $\mu_{0}=\mu(z=0)$, and it becomes especially simple to express the integrals over the azimuthal angle $\theta$ as a sum of contributions from angles (or wavenumbers) that do not and that do have a critical level, respectively. Assuming that the variation $\psi$ with height is monotonic and that the wind does not turn by more than $\pi$ from the surface up to the level under consideration (so that there is not more than one critical level per wavenumber), (12) becomes

$$
\begin{aligned}
M_{x}= & 8 \pi^{2} \rho_{0} N a b \mu_{0} h_{0}^{2} \int_{0}^{+\infty} \kappa^{2}\left|\hat{h}^{\prime}\right|^{2} d \kappa\left\{\cos \psi_{0}\left[I_{1}(z)-I_{2}(z)\right]\right. \\
& \left.+\sin \psi_{0}\left[I_{3}(z)-I_{4}(z)\right]\right\} \\
M_{y}= & 8 \pi^{2} \rho_{0} N a b \mu_{0} h_{0}^{2} \gamma \int_{0}^{+\infty} \kappa^{2}\left|\hat{h}^{\prime}\right|^{2} d \kappa\left\{\cos \psi_{0}\left[I_{3}(z)-I_{4}(z)\right]\right. \\
& \left.+\sin \psi_{0}\left[I_{5}(z)-I_{6}(z)\right]\right\}
\end{aligned}
$$

where the integrals $I_{1}-I_{6}$ are defined in Appendix A (the procedure to relax the limitations mentioned above will be described in section 2.3). These integrals must, in general, be calculated numerically, but at $z=0$, where $\psi=$ $\psi_{0}$ and the momentum flux $\left(M_{x}, M_{y}\right)$ is equal to the surface drag $\left(D_{x}, D_{y}\right)$, $I_{2}, I_{4}$ and $I_{6}$ vanish, the exponential in $I_{1}, I_{3}$ and $I_{5}$ equals 1 , and the limits of integration of these latter integrals may, without loss of generality, be taken to be 0 and $\pi$. If one further assumes that the mean vertical wind shear is 
zero, then $S(\theta, z)=0$, so the surface drag becomes

$$
\begin{aligned}
& D_{0 x}=16 \pi^{2} \rho_{0} N a b \mu_{0} h_{0}^{2} \cos \psi_{0} \int_{0}^{+\infty} \kappa^{2}\left|\hat{h}^{\prime}\right|^{2} d \kappa \int_{0}^{\pi / 2} \frac{\cos ^{2} \theta}{\left(\cos ^{2} \theta+\gamma^{2} \sin ^{2} \theta\right)^{1 / 2}} d \theta, \\
& D_{0 y}=16 \pi^{2} \rho_{0} N a b \mu_{0} h_{0}^{2} \gamma \sin \psi_{0} \int_{0}^{+\infty} \kappa^{2}\left|\hat{h}^{\prime}\right|^{2} d \kappa \int_{0}^{\pi / 2} \frac{\sin ^{2} \theta}{\left(\cos ^{2} \theta+\gamma^{2} \sin ^{2} \theta\right)^{1 / 2}} d \theta,
\end{aligned}
$$

where the integrals over $\theta$ in (15) and (16) result only from $I_{1}$ and $I_{5}$, because $I_{3}$ vanishes by symmetry when $S(\theta, z)=0$. These equations can be expressed in terms of the functions $G, B(\gamma)$ and $C(\gamma)$, defined by Phillips [23], as

$$
D_{0 x}=\rho_{0} N U_{0} b h_{0}^{2} G B(\gamma), \quad D_{0 y}=\rho_{0} N V_{0} b h_{0}^{2} G C(\gamma)
$$

where

$$
\begin{aligned}
G & =16 \pi^{2} \int_{0}^{+\infty} \kappa^{2}\left|\hat{h}^{\prime}(\kappa)\right|^{2} d \kappa \\
B(\gamma) & =\int_{0}^{\pi / 2} \frac{\cos ^{2} \theta}{\left(\cos ^{2} \theta+\gamma^{2} \sin ^{2} \theta\right)^{1 / 2}} d \theta \\
C(\gamma) & =\gamma^{2} \int_{0}^{\pi / 2} \frac{\sin ^{2} \theta}{\left(\cos ^{2} \theta+\gamma^{2} \sin ^{2} \theta\right)^{1 / 2}} d \theta
\end{aligned}
$$

(cf. [16]).

Taking into account (15)-(17), it may be shown that in (13)-(14) $\left(M_{x}, M_{y}\right)$ may be expressed in terms of $\left(D_{0 x}, D_{0 y}\right)$ in the following way:

$$
\begin{aligned}
& M_{x}=\frac{D_{0 x}}{2 B(\gamma)}\left[I_{1}(z)-I_{2}(z)\right]+\frac{\gamma D_{0 y}}{2 C(\gamma)}\left[I_{3}(z)-I_{4}(z)\right] \\
& M_{y}=\frac{\gamma D_{0 x}}{2 B(\gamma)}\left[I_{3}(z)-I_{4}(z)\right]+\frac{\gamma^{2} D_{0 y}}{2 C(\gamma)}\left[I_{5}(z)-I_{6}(z)\right],
\end{aligned}
$$

which reveal that the coefficients multiplying $D_{0 x}$ and $D_{0 y}$ do not depend on the detailed shape of the orography (as long as this has an elliptical horizontal 
cross-section) apart from its aspect ratio $\gamma$. This extends a similar result of Teixeira and Miranda [16] for the surface drag to the momentum flux. When $z=0,(21)-(22)$ reduce to

$$
\begin{aligned}
& M_{x}(z=0)=D_{x}=\frac{D_{0 x}}{2 B(\gamma)} I_{1}(z=0)+\frac{\gamma D_{0 y}}{2 C(\gamma)} I_{3}(z=0) \\
& M_{y}(z=0)=D_{y}=\frac{\gamma D_{0 x}}{2 B(\gamma)} I_{3}(z=0)+\frac{\gamma^{2} D_{0 y}}{2 C(\gamma)} I_{5}(z=0) .
\end{aligned}
$$

This is equivalent to Equation (1) of Miranda et al. [22] if the integrals $I_{1}(z=0), I_{3}(z=0)$ and $I_{5}(z=0)$ are expressed in terms of the functions $\alpha(\gamma)$ and $\beta(\gamma)$ defined by Teixeira and Miranda [16] (which in turn can be expressed in terms of $B(\gamma)$ and $C(\gamma)$ and $\gamma$ explicitly), and the effect of the terms involving the vertical derivatives of $(U, V)$ is put in evidence (see details in [16]).

\subsection{Non-monotonic turning wind angles and turning by more than $\pi$}

The expressions for the momentum flux derived above assume that the wind turning angle $\psi$ varies monotonically and that its total variation never exceeds $\pi$. In the real atmosphere, these conditions may not be met, so a strategy to deal with this limitation is outlined next.

The linear relation between both $\left(M_{x}, M_{y}\right)$ and $\left(D_{0 x}, D_{0 y}\right)$, and $\left(D_{x}, D_{y}\right)$ and $\left(D_{0 x}, D_{0 y}\right)$, expressed by (21)-(24), enables one to alternatively express the momentum flux in the following form

$$
\begin{aligned}
& M_{x}=C_{1} M_{x}(z=0)+C_{2} M_{y}(z=0), \\
& M_{y}=C_{3} M_{x}(z=0)+C_{4} M_{y}(z=0),
\end{aligned}
$$

where

$$
C_{1}=\frac{I_{3}(z=0)\left[I_{3}(z)-I_{4}(z)\right]-I_{5}(z=0)\left[I_{1}(z)-I_{2}(z)\right]}{I_{3}^{2}(z=0)-I_{1}(z=0) I_{5}(z=0)}
$$




$$
\begin{aligned}
C_{2} & =\frac{1}{\gamma} \frac{I_{3}(z=0)\left[I_{1}(z)-I_{2}(z)\right]-I_{1}(z=0)\left[I_{3}(z)-I_{4}(z)\right]}{I_{3}^{2}(z=0)-I_{1}(z=0) I_{5}(z=0)}, \\
C_{3} & =\gamma \frac{I_{3}(z=0)\left[I_{5}(z)-I_{6}(z)\right]-I_{5}(z=0)\left[I_{3}(z)-I_{4}(z)\right]}{I_{3}^{2}(z=0)-I_{1}(z=0) I_{5}(z=0)}, \\
C_{4} & =\frac{I_{3}(z=0)\left[I_{3}(z)-I_{4}(z)\right]-I_{1}(z=0)\left[I_{5}(z)-I_{6}(z)\right]}{I_{3}^{2}(z=0)-I_{1}(z=0) I_{5}(z=0)} .
\end{aligned}
$$

Although (25)-(26) describe the momentum flux at a generic level $z$ as a function of its value at the surface $z=0$, nothing prevents us from extending this relation to multiple layers, with different bottom heights.

Hence to deal with the effects of either a non-monotonic wind profile or a wind turning angle exceeding $\pi$, one must split the atmosphere into various layers where the wind is monotonic and does not turn by more than $\pi$. At the level where $d \psi / d z$ changes sign, or the difference $\left|\psi-\psi_{0}\right|$ exceeds $\pi$ (whichever happens first), a new layer must begin where (25)-(26) is applied, replacing $(z=0)$ by the height of the bottom of the new layer. This procedure, which may be carried out for as many layers as necessary, allows us to express the momentum flux within each layer as a function of the momentum flux as its bottom, which is equal to the momentum flux at the top of the layer below, calculated previously. This is especially relevant at low Richardson numbers, such as envisaged here, because the momentum flux is then only partially absorbed at critical levels, and what happens above them (i.e. whatever additional variation of $\left(M_{x}, M_{y}\right)$ takes place) matters. This is not a concern at high $R i$, since the momentum flux is in that case totally absorbed at the first critical level encountered by a given wavenumber, hence the momentum flux simply vanishes at that wavenumber above that height. 


\subsection{The momentum flux divergence}

The quantity that is most relevant to drag parametrization, since it corresponds to the acceleration directly imparted to the mean flow, is the wave momentum flux divergence, i.e. the vertical derivative of (21)-(22). When these equations are differentiated, since they depend on the single integrals over $\theta$ expressed by $I_{1}-I_{6}$, they take a closed analytical form. It turns out that, depending on whether $d \psi / d z$ is positive or negative, this momentum flux divergence takes a different sign. The final expressions, valid for an arbitrary sign of $d \psi / d z$, and accurate to third-order in the small parameter $\varepsilon$ used in the WKB approximation, are

$$
\begin{aligned}
\frac{d M_{x}}{d z}(z)= & \frac{1}{2}\left|\frac{d \psi}{d z}\right| \frac{\sin \psi}{\left(\sin ^{2} \psi+\gamma^{2} \cos ^{2} \psi\right)^{1 / 2}}\left[1-S_{\psi}(z)\right] e^{S_{\psi}(z)-S_{\psi}(z=0)} \\
& \times\left[1+e^{-2 \pi C_{\psi}(z)}\right]\left[\frac{\gamma D_{0 y}}{C(\gamma)} \cos \psi-\frac{D_{0 x}}{B(\gamma)} \sin \psi\right] \\
\frac{d M_{y}}{d z}(z)= & \frac{1}{2}\left|\frac{d \psi}{d z}\right| \frac{\cos \psi}{\left(\sin ^{2} \psi+\gamma^{2} \cos ^{2} \psi\right)^{1 / 2}}\left[1-S_{\psi}(z)\right] e^{S_{\psi}(z)-S_{\psi}(z=0)} \\
& \times\left[1+e^{-2 \pi C_{\psi}(z)}\right]\left[\frac{\gamma D_{0 x}}{B(\gamma)} \sin \psi-\frac{\gamma^{2} D_{0 y}}{C(\gamma)} \cos \psi\right]
\end{aligned}
$$

where

$$
\begin{aligned}
S_{\psi}(z)= & \frac{1}{8} \frac{\left(U^{\prime} \sin \psi-\gamma V^{\prime} \cos \psi\right)^{2}}{N^{2}\left(\sin ^{2} \psi+\gamma^{2} \cos ^{2} \psi\right)} \\
& +\frac{1}{4} \frac{(U \sin \psi-\gamma V \cos \psi)\left(U^{\prime \prime} \sin \psi-\gamma V^{\prime \prime} \cos \psi\right)}{N^{2}\left(\sin ^{2} \psi+\gamma^{2} \cos ^{2} \psi\right)} \\
C_{\psi}(z)= & \frac{N\left(\sin ^{2} \psi+\gamma^{2} \cos ^{2} \psi\right)^{1 / 2}}{\left|U^{\prime} \sin \psi-\gamma V^{\prime} \cos \psi\right|}\left[1-\frac{1}{8} \frac{\left(U^{\prime} \sin \psi-\gamma V^{\prime} \cos \psi\right)^{2}}{N^{2}\left(\sin ^{2} \psi+\gamma^{2} \cos ^{2} \psi\right)}\right]
\end{aligned}
$$

Note that $S_{\psi}=S(\theta=\psi \pm \pi / 2, z)$ and $C_{\psi}=C(\theta=\psi+\pi / 2)$, which means that, although $C(\theta)$ was not a function of $z, C_{\psi}$ becomes one, by being evaluated at a $\theta$ that depends on $z$ (through $\psi$ ). On the other hand, in $C_{\psi}$, 
$\left(U_{c}^{\prime}, V_{c}^{\prime}\right)$ has been replaced by $\left(U^{\prime}, V^{\prime}\right)$ because the transformation between $C(\theta)$ and $C_{\psi}(z)$ already takes into account the fact that $\left(U^{\prime}, V^{\prime}\right)$ is taken at the critical level. It may easily be confirmed that (31)-(32) satisfy (5), as required.

Equations (31)-(32) express $d\left(M_{x}, M_{y}\right) / d z$ as a function of the surface drag in the absence of shear $\left(D_{0 x}, D_{0 y}\right)$. Again, when multiple critical levels exist (i.e., the wind has turned by an angle larger than $\pi$ ), these equations cannot be applied directly above the level where that occurs. Then, a procedure similar to the one outlined for the momentum flux must be followed. First of all, using (23)-(24), $d M_{x} / d z$ and $d M_{y} / d z$ must be expressed as functions of $M_{x}(z=0)$ and $M_{y}(z=0)$, namely

$$
\begin{aligned}
\frac{d M_{x}}{d z}(z)= & \frac{1}{2}\left|\frac{d \psi}{d z}\right| \frac{\sin \psi}{\left(\sin ^{2} \psi+\gamma^{2} \cos ^{2} \psi\right)^{1 / 2}}\left[1-S_{\psi}(z)\right] e^{S_{\psi}(z)-S_{\psi}(z=0)} \\
& \times\left[1+e^{-2 \pi C_{\psi}(z)}\right]\left(C_{5} M_{x}(z=0)+C_{6} M_{y}(z=0)\right) \\
\frac{d M_{y}}{d z}(z)= & -\frac{1}{2} \gamma\left|\frac{d \psi}{d z}\right| \frac{\cos \psi}{\left(\sin ^{2} \psi+\gamma^{2} \cos ^{2} \psi\right)^{1 / 2}}\left[1-S_{\psi}(z)\right] e^{S_{\psi}(z)-S_{\psi}(z=0)} \\
& \times\left[1+e^{-2 \pi C_{\psi}(z)}\right]\left(C_{5} M_{x}(z=0)+C_{6} M_{y}(z=0)\right)
\end{aligned}
$$

where

$$
\begin{aligned}
C_{5} & =2 \frac{I_{3}(z=0) \cos \psi+I_{5}(z=0) \sin \psi}{I_{3}^{2}(z=0)-I_{1}(z=0) I_{5}(z=0)} \\
C_{6} & =-\frac{2}{\gamma} \frac{I_{1}(z=0) \cos \psi+I_{3}(z=0) \sin \psi}{I_{3}^{2}(z=0)-I_{1}(z=0) I_{5}(z=0)} .
\end{aligned}
$$

Secondly, this approach must be applied to successive layers, taking $\left[M_{x}(z=\right.$ $0), M_{y}(z=0)$ ] to correspond to the bottom of each layer. This implies that, when more than one layer exists, calculating the momentum flux divergence requires also calculating the momentum flux, but fortunately it is only necessary to do this at the discrete levels delimiting the various layers. 


\section{Linear numerical model}

The linear numerical model that will be used to assess the limitations of the WKB approximation follows a similar approach to that presented in appendix A of Teixeira and Miranda [16], which was adapted from a model originally developed by Sivertsen [24]. The basic idea will be sketched next, but the reader is referred to [16] for more details.

Without loss of generality, the solution to the hydrostatic Taylor-Goldstein equation,

$$
\hat{w}^{\prime \prime}+\left[\frac{N^{2}\left(k^{2}+l^{2}\right)}{(U k+V l)^{2}}-\frac{U^{\prime \prime} k+V^{\prime \prime} l}{U k+V l}\right] \hat{w}=0
$$

(where $\hat{w}$ is the Fourier transform of the vertical velocity perturbation), may be expressed as the product of an amplitude and a complex exponential involving the phase,

$$
\hat{w}=\hat{w}(z=0) \exp \left[i \int_{0}^{z} m(\xi) d \xi\right] .
$$

In this expression, which does not assume the WKB approximation, $m$ is the vertical wavenumber of the mountain waves and the amplitude $\hat{w}(z=0)$ is determined by the lower boundary condition,

$$
\hat{w}(z=0)=i\left(U_{0} k+V_{0} l\right) \hat{h}
$$

which expresses the fact that the flow is tangential to the orography at the surface. When (40) is introduced into (39), an equation for $m$ results:

$$
i m^{\prime}-m^{2}+\frac{N^{2}\left(k^{2}+l^{2}\right)}{(U k+V l)^{2}}-\frac{U^{\prime \prime} k+V^{\prime \prime} l}{U k+V l}=0 .
$$

Despite being nonlinear, compared with the linear Taylor-Goldstein equation, (42) has the advantage of being a first-order differential equation, requiring 
the specification of only one boundary condition. This equation is solved here in Fourier space for a large set of horizontal wavenumber vectors $\mathbf{k}=(k, l)$.

For $\mathbf{k}$ with its critical level $z_{c}$ lying inside the computational domain, i.e. for $0<z_{c}<H$, where $H$ is the top of computational domain, the calculation starts from the vicinity of the critical level towards either the top or the bottom of the domain. However, since the vertical wave number $m$ diverges to infinity near a critical level, a more convenient approach is solving for the inverse of $m, L=1 / m$ (cf. [16]). It can be shown from (42) that the equation satisfied by $L$ is

$$
L^{\prime}=i\left\{1-\left[\frac{N^{2}\left(k^{2}+l^{2}\right)}{(U k+V l)^{2}}-\frac{U^{\prime \prime} k+V^{\prime \prime} l}{U k+V l}\right] L^{2}\right\} .
$$

The behaviour of $L$ in the vicinity of critical levels is determined using the Frobenius solution (see Grubišić amd Smolarkiewicz [19]), which is expressed by equation (A.5) in appendix $\mathrm{A}$ of [16]:

$$
L=\frac{z-z_{c}}{-\frac{i}{2} \pm\left[\frac{N^{2}\left(k^{2}+l^{2}\right)}{\left(U_{c}^{\prime} k+V_{c}^{\prime} l\right)^{2}}-\frac{1}{4}\right]^{1 / 2}},
$$

where the sign in the denominator is determined by the condition that the wave energy propagates upward. This solution provides the boundary condition (initial value) for (43), so that the numerical method can be implemented.

For one of the idealized wind profiles to be considered (wind that turns with height (48)), if the computational domain is defined such that the wind spans an angle of $\pi$ from its bottom to its top, any wavenumber $\mathbf{k}$ has exactly one critical level within the domain, so the above approach can be used to determine all the required solutions. However, for the other wind 
profile to be considered (linear wind profile (47)), as the shear is constant, some horizontal wavenumbers $\mathbf{k}=(k, l)$ have their critical levels $z_{c}$ lying outside the computational domain. For such wavenumbers, two strategies are employed. If the critical level occurs relatively near to the domain, i.e. $D=\min \left(\left|z_{c}-H\right|,\left|z_{c}-0\right|\right) \leq R$ where $R$ is some positive threshold value, then the solution process still starts from the critical level, but only values of $m$ (or $L$ ) within the domain are recorded. If the critical level is located far away from the computational domain, i.e. $D>R$, then the boundary condition applied at the top of the domain assumes that the vertical wave number $m$ reached a constant value, i.e. $m^{\prime}=0$. Since, for a linear wind profile, $\left(U^{\prime \prime}, V^{\prime \prime}\right)=0$, (42) then implies that the boundary condition at the top of the domain is

$$
m(z=H)=\frac{N\left(k^{2}+l^{2}\right)^{1 / 2}}{U(z=H) k+V(z=H) l},
$$

where (45) corresponds to upward wave energy propagation.

The numerical scheme employed to solve (43) is a fourth-order RungeKutta method with sufficiently fine spacing between grid-points throughout the domain (20000 grid points), and the threshold value $R$ is set to be $3 H$. With the vertical wave number $m$ obtained numerically, the Fourier transform of the vertical velocity perturbation $\hat{w}$ can be numerically integrated using (40). Furthermore, from equations (14)-(16) of Teixeira and Miranda [17], the momentum flux can be rewritten as

$$
\left(M_{x}, M_{y}\right)=4 \pi^{2} \rho_{0} \int_{-\infty}^{+\infty} \int_{-\infty}^{+\infty} \frac{(k, l)}{k^{2}+l^{2}} \operatorname{Re}(m)|\hat{w}|^{2} d k d l .
$$

The above integral is transformed using the polar elliptical coordinate system introduced before (6). The integration (over $z$ for $\hat{w}$ and over $\theta$ for 
$\left.\left(M_{x}, M_{y}\right)\right)$ is performed numerically using finite summation with sufficiently small (uniform) intervals $\Delta z$ and $\Delta \theta(\Delta z=H / 20000$ and $\Delta \theta=2 \pi / 1007)$. The numerical results obtained through this method will be compared next with those calculated using the WKB approximation.

\section{Results}

Following Teixeira and Miranda [17], the theoretical model described in section 2 will be assessed for idealized wind profiles where the Richardson number is constant. This reduces the number of parameters on which the results depend to a minimum, and serves the purpose of testing the accuracy of the WKB approximation at relatively low $R i$. These profiles do not require the calculation of the momentum flux in various layers, as they are monotonic, and we are concerned only with the region where they turn by angles lower than $\pi$. These results will be compared with those produced by the linear numerical model described in the previous section, and contrasted with equivalent results for flow over an axisymmetric mountain [17].

\subsection{Linear wind profile}

The first wind profile to be considered is one of the simplest wind profiles with constant $R i$ and directional shear:

$$
U=U_{0} \cos \delta-\alpha z \sin \left(\delta+\frac{\pi}{4}\right), \quad V=U_{0} \sin \delta+\alpha z \cos \left(\delta+\frac{\pi}{4}\right),
$$

where $U_{0}$ is the wind speed at the surface, $\alpha$ is the magnitude of the shear rate (assumed to be constant) and $\delta$ is the angle between the surface wind velocity and the $x$ direction (and therefore also with the main axes of the orography - see Fig. 1). Note that, from (47), $R i=N^{2} / \alpha^{2}$. For $\delta=\pi / 4$ (47) 


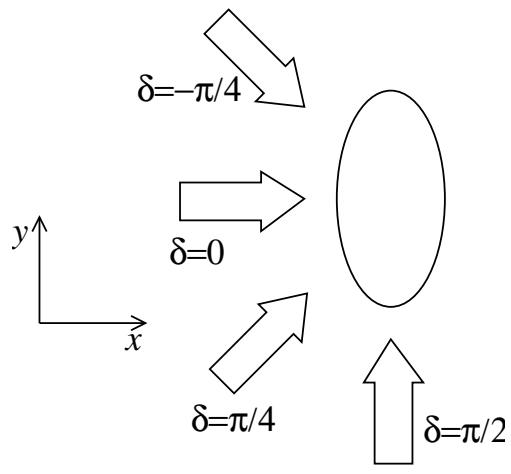

Figure 1: Schematic diagram of the 4 situations to be considered in Figs. 3 and 8, 4 and 9, 5 and 10, and 6 and 11, corresponding to a surface wind (block arrows) that makes an angle of $\delta=0, \delta=\pi / 2, \delta=\pi / 4$ and $\delta=-\pi / 4$ with the $x$ direction, respectively. The ellipse denotes a contour of surface elevation associated with the mountain.

reduces to Eq. (49) of [17], with the difference that $U_{0}$ is rescaled by a factor of $\sqrt{2}$. While describing a shear misaligned with the surface wind by an angle of $\pi / 4$, as Eq. (49) of [17], (47) additionally allows this wind profile to be rotated by any desired angle $\delta$, which is important for attaining maximum generality in flow over anisotropic orography. As pointed out by Teixeira and Miranda [17], this wind profile (illustrated in Fig. 2 for $\delta=\pi / 4$ ) has the advantage over other linearly varying wind profiles that the angle spanned by the wind velocity over the whole atmosphere (i.e. $0<z<+\infty$ ) is $3 \pi / 4$, which is relatively close to $\pi$, hence a large fraction of all wavenumbers have a critical level. As shown by Teixeira et al. [11], when this happens the impact of the wind profile above the critical levels is minimized, even for relatively low $R i$, making this an ideal test case. The forms taken by $S(\theta, z), C(\theta)$, $S_{\psi}(z)$ and $C_{\psi}(z)$ for this case are listed in Appendix B.

As in [23] and [16], flow over orography with $\gamma=0.5$ is considered. This 
corresponds to a substantially anisotropic mountain, which nevertheless does not approach a 2D geometry. Four values of $\delta$ are considered (see Fig. 1): $\delta=0$, corresponding to a surface wind perpendicular to the major axis of the mountain (Fig. 3), $\delta=\pi / 2$, corresponding to a surface wind parallel to the major axis of the mountain (Fig. 4), and $\delta= \pm \pi / 4$, corresponding to oblique surface winds (Figs. 5 and 6). These values of $\delta$ were chosen because the behaviour of the momentum flux depends on the angles between the major axis of the mountain and both the wind velocity and the shear rate. The four selected values of $\delta$ span all possible distinct combinations for which $\delta$ is a multiple of $\pi / 4$.

In Figs. 3-6, the thick red lines and symbols correspond to $\gamma=0.5$, whereas the thin blue lines and symbols correspond to $\gamma=1$ (the situation considered in [17]) for comparison. The solid lines denote results from WKB theory, the circles denote results from the linear numerical model (which does not assume the WKB approximation) and the dotted lines correspond to the limit $R i \rightarrow \infty$. In each of these figures, panels (a), (c) and (e) show the $x$ component of the momentum flux, while panels (b), (d) and (f) show the $y$ component. In panels (a) and (b), $R i=5$, in panels (c) and (d), $R i=1$, and in panels (e) and (f), $R i=0.5$. In Figs. 3-6 the momentum flux has been normalized so as to take a value of 1 at the surface for $R i \rightarrow \infty$ whenever possible, and zero otherwise. This means that both $M_{x}$ and $M_{y}$ are normalized by $D_{0 x}$ when $\delta=0$ (because $D_{0 y}=0$ ), and both $M_{x}$ and $M_{y}$ are normalized by $D_{0 y}$ when $\delta=\pi / 2$ (because $D_{0 x}=0$ ), but $M_{x}$ is normalized by $D_{0 x}$ and $M_{y}$ by $D_{0 y}$ when $\delta= \pm \pi / 4$. This choice, which follows [14], [16] and [17], allows us to focus solely on wind shear and mountain geometry 


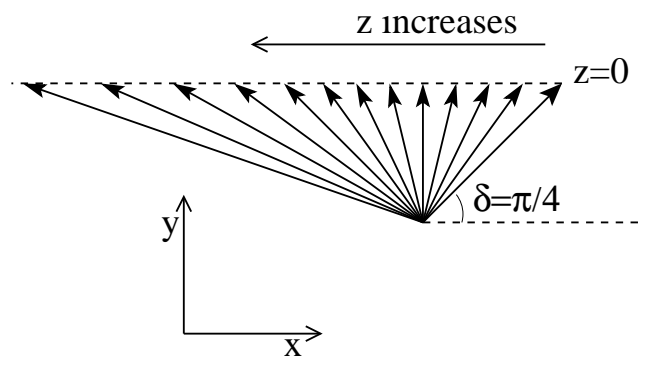

Figure 2: Schematic diagram of the linear wind profile (47) for $\delta=\pi / 4$. Arrows denote the wind velocity at different heights. Note that, in accordance with (47), the vertical shear is at an angle of $\pi / 4$ to the surface wind.

effects when analyzing the behaviour of the momentum flux.

In Fig. 3 it can be seen that, when $\delta=0, M_{x}$ decreases faster with height and $M_{y}$ takes lower values for $\gamma=0.5$ than for $\gamma=1$. Fig. 4 shows that when $\delta=\pi / 2$, while $M_{y}$ is enhanced (in absolute value) for $\gamma=0.5$ relative to $\gamma=1, M_{y}$ decreases more slowly with height. In Fig. 5, on the other hand, it can be seen that when $\delta=\pi / 4$, both $M_{x}$ and $M_{y}$ vary faster with height when $\gamma=0.5$ than when $\gamma=1$, and the maximum attained by $M_{y}$ is enhanced. Finally, Fig. 6 shows that when $\delta=-\pi / 4$ both $M_{x}$ and $M_{y}$ vary with height more slowly when $\gamma=0.5$ than when $\gamma=1$. The vertical dotted lines in Figs. 3 and 5 , which denote the heights where $U=0$, and the vertical dashed lines in Figs. 4 and 6, which denote the heights where $V=0$, help us to interpret this behaviour. Since the variation of the momentum flux with height is due to critical levels, $M_{x}$ should suffer a major depletion at the heights where $U=0$ and $M_{y}$ similarly at the heights where $V=0$ (which indeed happens). The reason why this variation is faster in the first than in the second case can be explained by the fact that, for $\gamma=0.5$, proportionally more wave energy exists at wavenumbers 

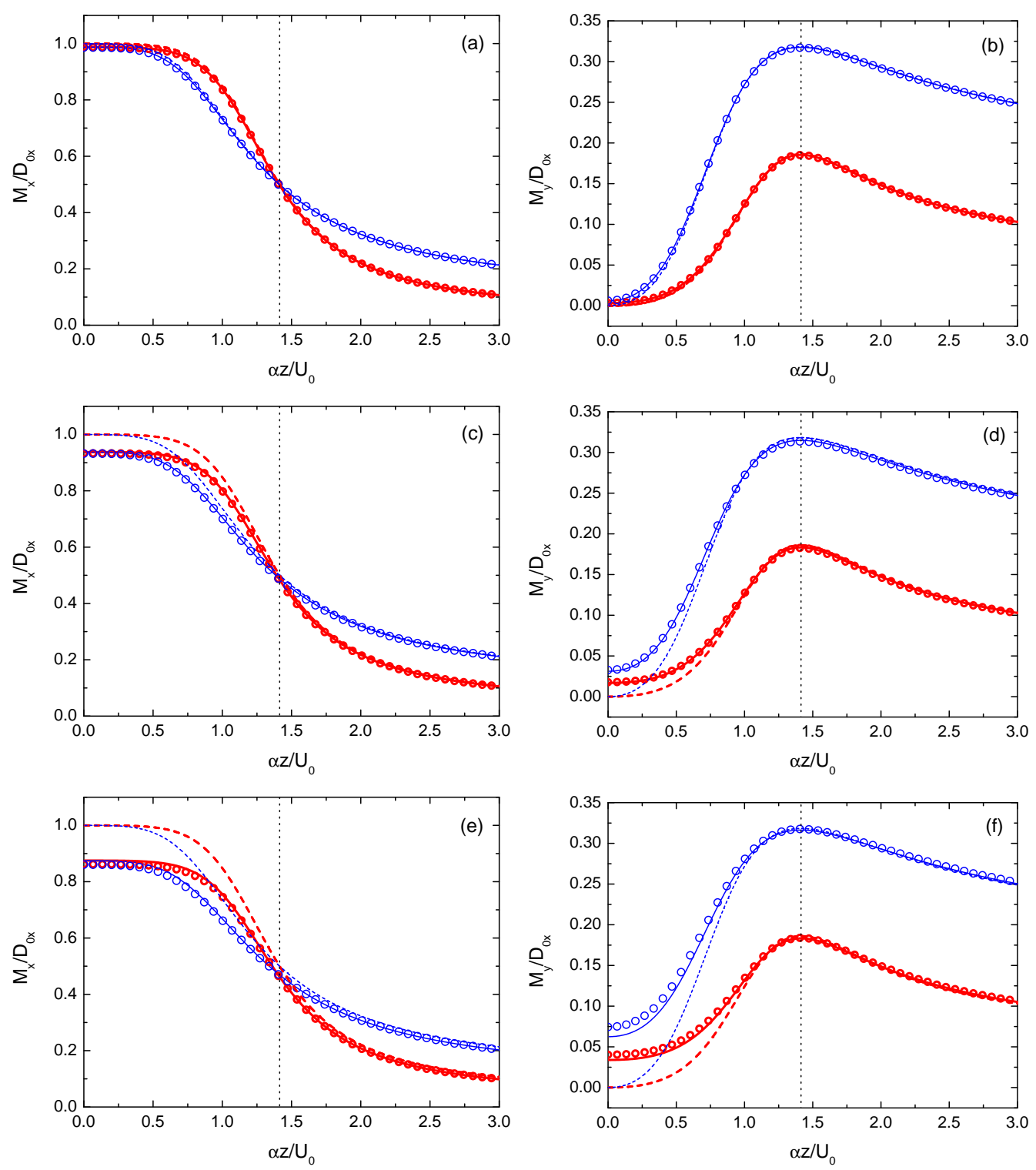

Figure 3: Normalized momentum flux as a function of normalized height for the wind profile (47) with $\delta=0$. Thick red lines and symbols: $\gamma=0.5$, thin blue lines and symbols: $\gamma=1$. Solid lines: WKB approximation, dotted lines: $R i \rightarrow \infty$ limit, circles: linear numerical model. The vertical dotted line denotes the height where $U=0\left(\alpha z / U_{0}=\sqrt{2}\right)$. (a) $M_{x} / D_{0 x}$ for $R i=5$, (b) $M_{y} / D_{0 x}$ for $R i=5$, (c) $M_{x} / D_{0 x}$ for $R i=1$, (d) $M_{y} / D_{0 x}$ for $R i=1$, (e) $M_{x} / D_{0 x}$ for $R i=0.5$, (f) $M_{y} / D_{2.5}$ for $R i=0.5$. 

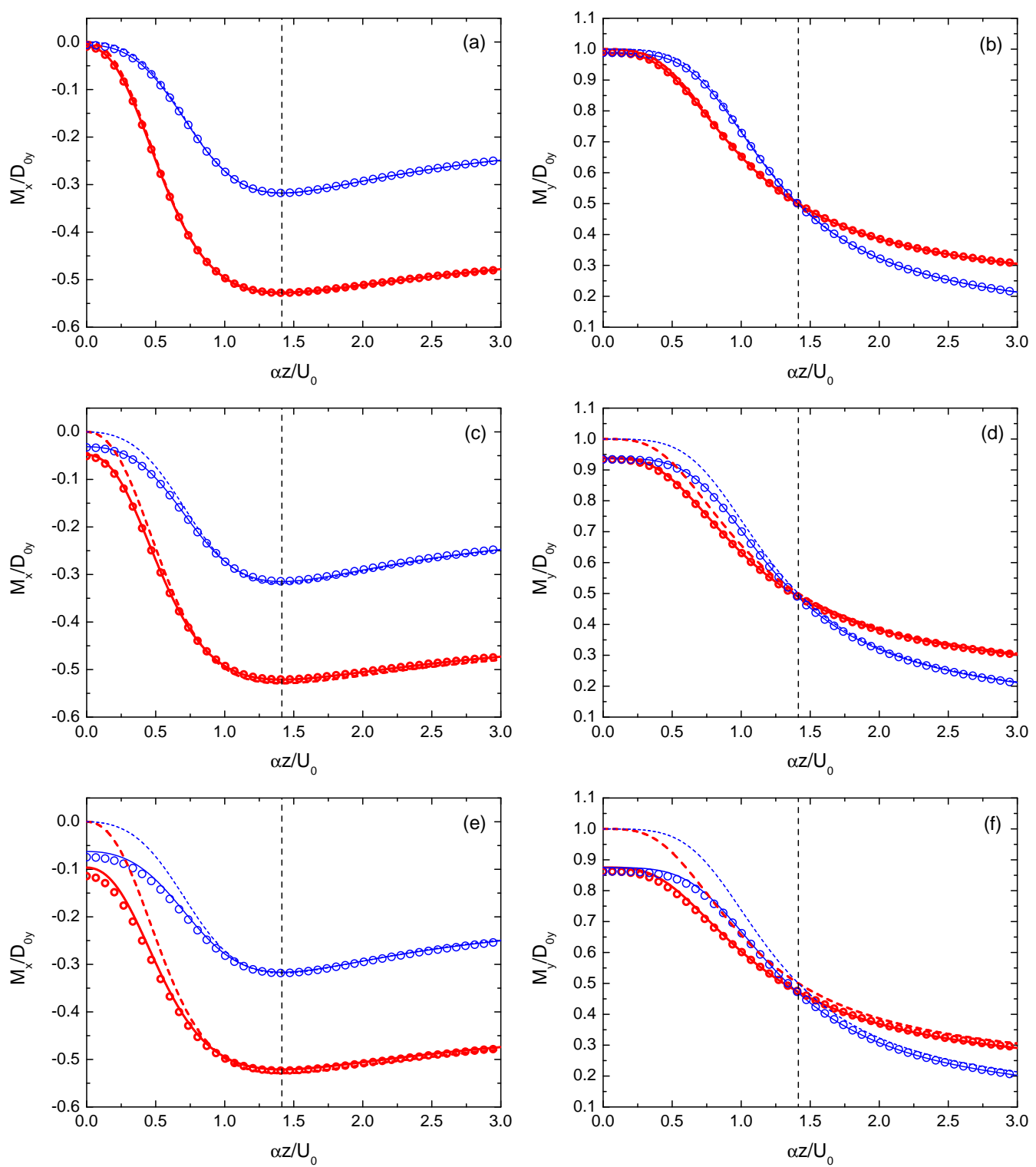

Figure 4: Similar to Fig. 3, but for $\delta=\pi / 2$. The vertical dashed line denotes the height where $V=0\left(\alpha z / U_{0}=\sqrt{2}\right)$. (a) $M_{x} / D_{0 y}$ for $R i=5$, (b) $M_{y} / D_{0 y}$ for $R i=5$, (c) $M_{x} / D_{0 y}$ for $R i=1$, (d) $M_{y} / D_{0 y}$ for $R i=1$, (e) $M_{x} / D_{0 y}$ for $R i=0.5$, (f) $M_{y} / D_{0 y}$ for $R i=0.5$. 

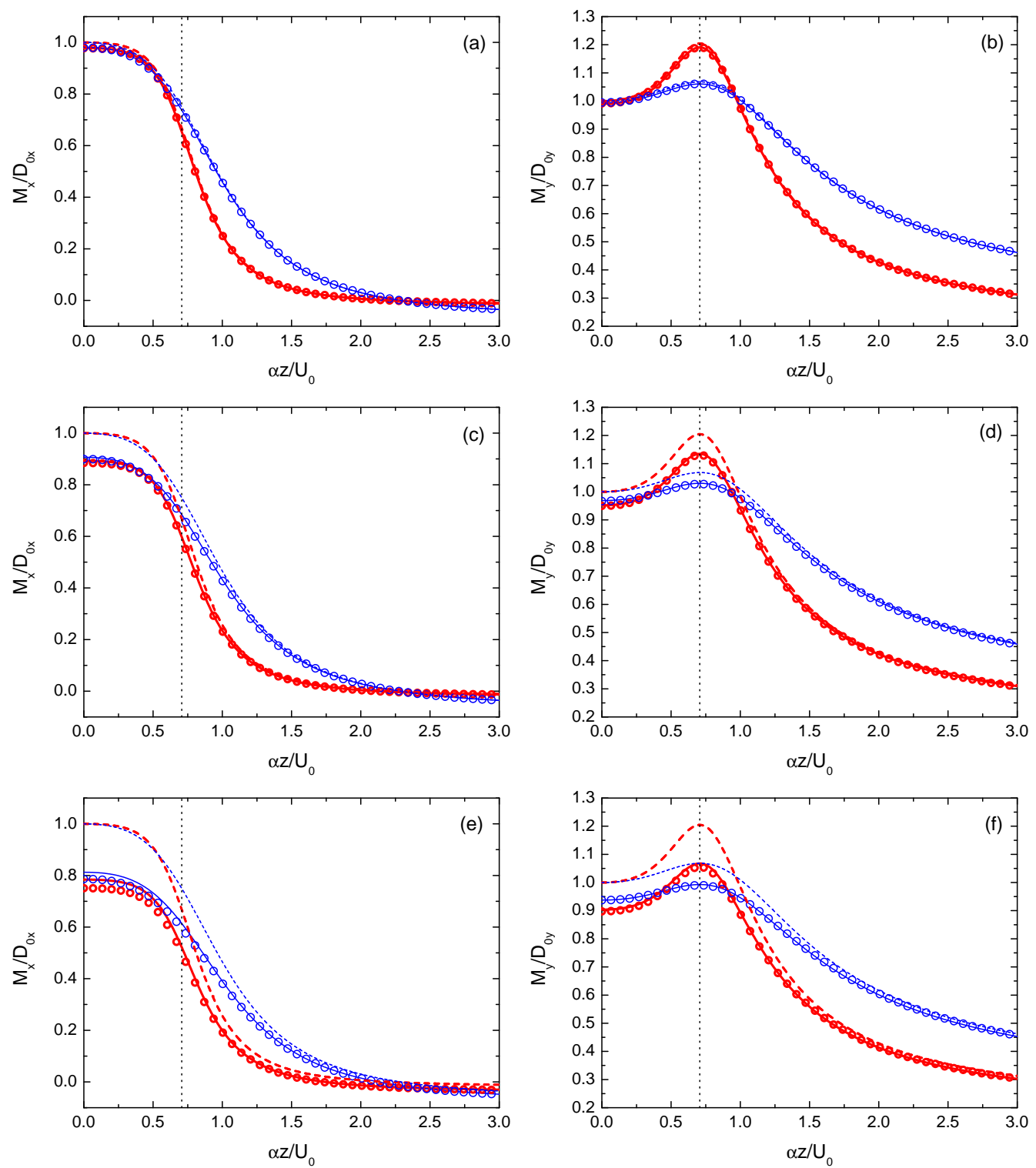

Figure 5: Similar to Fig. 3, but for $\delta=\pi / 4$. The vertical dotted line denotes the height where $U=0\left(\alpha z / U_{0}=\sqrt{2} / 2\right)$. (a) $M_{x} / D_{0 x}$ for $R i=5$, (b) $M_{y} / D_{0 y}$ for $R i=5$, (c) $M_{x} / D_{0 x}$ for $R i=1$, (d) $M_{y} / D_{0 y}$ for $R i=1$, (e) $M_{x} / D_{0 x}$ for $R i=0.5$, (f) $M_{y} / D_{0 y}$ for $R i=0.5$. 

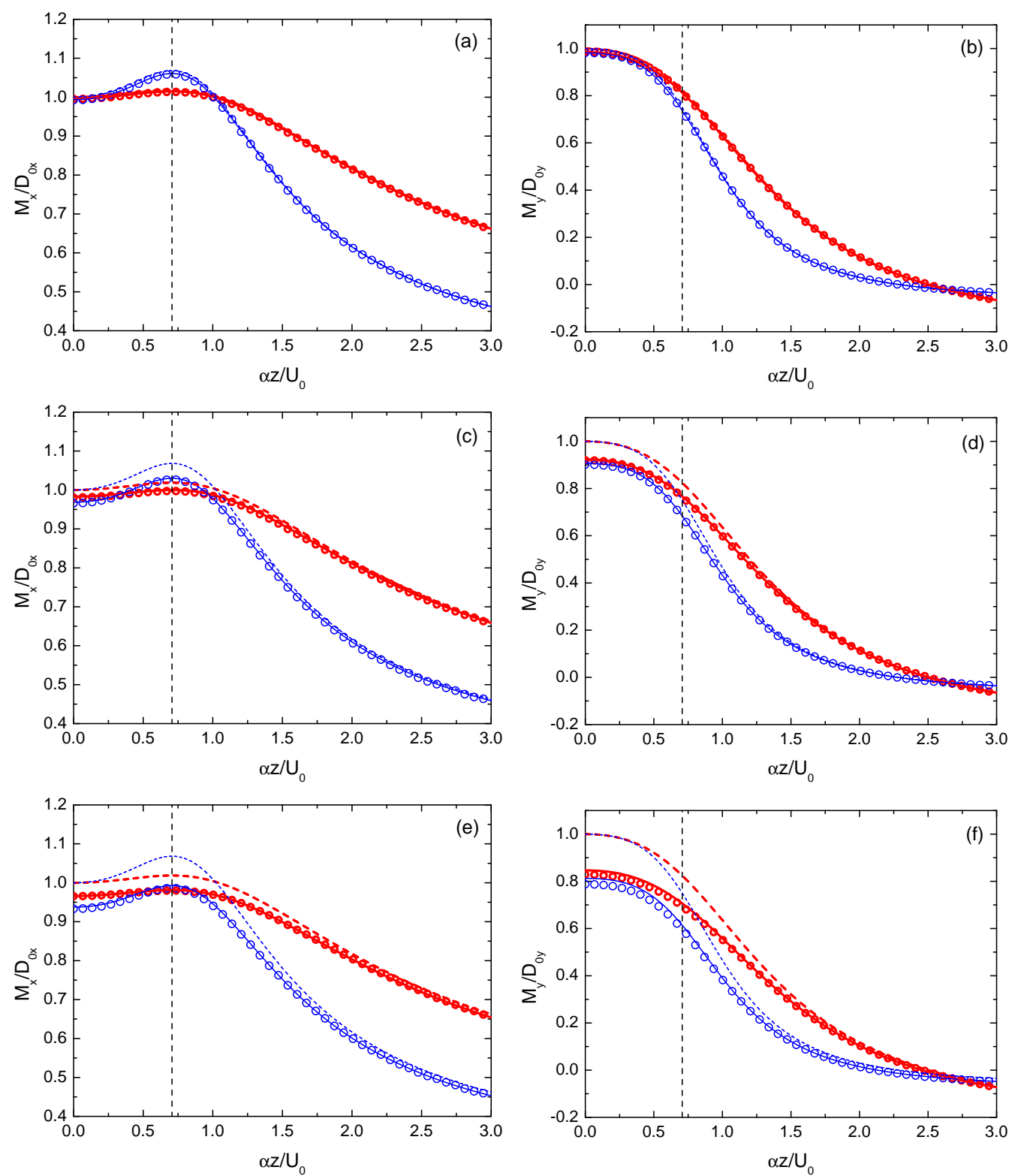

Figure 6: Similar to Fig. 3 , but for $\delta=-\pi / 4$. The vertical dashed line denotes the height where $V=0\left(\alpha z / U_{0}=\sqrt{2} / 2\right)$. (a) $M_{x} / D_{0 x}$ for $R i=5$, (b) $M_{y} / D_{0 y}$ for $R i=5$, (c) $M_{x} / D_{0 x}$ for $R i=1$, (d) $M_{y} / D_{0 y}$ for $R i=1$, (e) $M_{x} / D_{0 x}$ for $R i=0.5$, (f) $M_{y} / D_{0 y}$ for $R i=0.5$. Note that in this case $D_{0 y}<0$. 
perpendicular to the mountain (along $x$ ) than at wavenumbers parallel to the mountain (along $y$ ), in comparison to a situation where the mountain is axisymmetric $(\gamma=1)$. In the $2 \mathrm{D}$ orography limit $(\gamma=0)$, the variation of $M_{x}$ with height in Fig. 3, for example, would resemble a step function, as is well known to occur from Booker and Bretherton's pioneering study [18]. Note also how, in Figs. 3-6, the component of the momentum flux perpendicular to the wind component that vanishes at the signalled heights attains a maximum or minimum there. This follows from (5), since that equation can only be satisfied when one of the mean wind components is zero if the vertical derivative of the other component of the momentum flux is zero. However, (5) by itself does not impose any constraint on the component of the momentum flux in the direction of the wind component that vanishes. That aspect is determined by the dynamics of the mountain waves.

When $R i=5$, it can be seen in Figs. 3-6(a,b) that, both for $M_{x}$ and $M_{y}$, there is excellent agreement between WKB theory (solid lines) and the linear numerical model (circles). In turn, the corresponding lines and symbols differ very little from the dotted lines that denote the limit $R i \rightarrow \infty$. When $R i=1$ (Figs. 3-6(c,d)), both $M_{x}$ and $M_{y}$ decrease over the whole range of displayed heights, but especially near the surface, except in the case of Fig. 3(d), where $M_{y}$ actually increases near the surface. In all cases, this corresponds to weaker vertical gradients of $M_{x}$ and $M_{y}$, i.e. a lower momentum flux divergence. This is consistent with the behaviour of the drag at the surface, which is known to decrease as $R i$ decreases for linear wind profiles $[14,15,16]$. Agreement between WKB theory and the linear numerical model remains virtually perfect, suggesting that the WKB approximation is accu- 
rate. When $R i=0.5$ (Figs. 3-6(e,f)), $M_{x}$ and $M_{y}$ decrease further (except in Fig. 3(f)), departing more from the reference values of 1 or 0 taken at the surface for $R i \rightarrow \infty$. This corresponds to smaller gradients of $M_{x}$ and $M_{y}$, consistent with a lower surface drag. Agreement between WKB theory and the linear numerical model remains very good, but some slight departures start to emerge: for example, in Fig. 3(f) $M_{y}$ is slightly underestimated by WKB theory, and in Figs. 4(e) and 5(e) WKB theory instead slightly overestimates $M_{x}$. This latter result is consistent with the well-known fact that the surface drag is overestimated by WKB theory for linear wind profiles at low $R i[14,16]$. The fact that in Fig. $3(\mathrm{~d}, \mathrm{f}) M_{y}(z=0) \neq 0$, in $4(\mathrm{c}, \mathrm{e})$ $M_{x}(z=0) \neq 0$, and in Figs. $5(\mathrm{c}-\mathrm{f})$ or $6(\mathrm{c}-\mathrm{f}) M_{x}(z=0)$ and $M_{y}(z=0)$ do not decrease with $R i$ in the same proportion, means that the surface drag becomes misaligned with the surface wind. This aspect is well captured by WKB theory.

\subsection{Wind that turns with height}

The other wind profile to be considered describes a wind that turns with height at a constant rate keeping its magnitude constant [14, 16, 17],

$$
U=U_{0} \cos (\beta z+\delta), \quad V=U_{0} \sin (\beta z+\delta)
$$

where $\beta$ is a constant and $\delta$ has the same meaning as before. This wind profile is depicted in Fig. 7 for $\delta=\pi / 4$. Note that, from (48), $R i=N^{2} /\left(U_{0} \beta\right)^{2}$. Although for this wind profile $\psi$ monotonically increases with height (as for the previous one), multiple critical levels exist for $\beta z / \pi>1$. However, following [17], attention will be focused here only on the region of the flow near the surface where $\beta z / \pi \leq 1$. Since all higher derivatives of (48) with 
respect to $z$ are non-zero, this wind profile provides a more stringent test on the WKB approximation than (47). The forms taken by $S(\theta, z), C(\theta), S_{\psi}(z)$ and $C_{\psi}$ in the present case are shown in Appendix B.

Figs. 8, 9, 10 and 11 present the two components of the momentum flux, normalized in the same way as in the previous section, as a function of normalized height, for $\delta=0, \delta=\pi / 2, \delta=\pi / 4$ and $\delta=-\pi / 4$, respectively. The definition of quantities presented in each panel and the meaning of lines and symbols are entirely analogous to those introduced in the previous section for Figs. 3-6. In Fig. $8(\mathrm{a}, \mathrm{b})$ it can be seen that when $\delta=0$, $M_{x}$, although more constant at the bottom and top of the plotted domain, decreases faster at mid-levels for $\gamma=0.5$ than for $\gamma=1$. $M_{y}$, on the other hand, is considerably lower in the first case than in the second. For $\delta=\pi / 2$ (Fig. $9(\mathrm{a}, \mathrm{b})), M_{x}$ is lower (larger in absolute value) for $\gamma=0.5$ than for $\gamma=1$, and $M_{y}$ has a slower decrease with height. When $\delta=\pi / 4$ (Fig. $10(\mathrm{a}, \mathrm{b})), M_{x}$ decreases faster near the height where $U=0$, but $M_{y}$, besides having a more pronounced maximum, decreases more slowly near the height where $V=0$. Finally, when $\delta=-\pi / 4$ (Fig. 11(a,b)), $M_{x}$ again decreases faster at the height where $U=0$ for $\gamma=0.5$ than for $\gamma=1$, and $M_{y}$ decreases more slowly at the height where $V=0$ (but these two heights are exchanged relative to Fig. 10). This behaviour of the momentum flux resembles, to a certain extent (especially in Figs. 8 and 9), that described in the previous section, which is perhaps not surprising given that the wind profiles have some similarities (the surface wind makes the same angles with the orography, and the velocity turns counter-clockwise as height increases in both wind profiles). Differences from $\gamma=1$ are, again, justified by the 


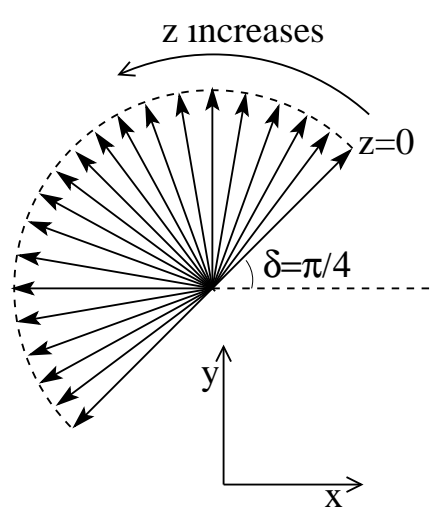

Figure 7: Schematic diagram of the wind that turns with height (48) for $\delta=\pi / 4$. Arrows denote the wind velocity at different heights.

fact that the orography launches a wave spectrum that has more energy at wavenumbers along $x$ than at wavenumbers along $y$. Hence, also here, at heights where $U=0, M_{x}$ varies faster when $\gamma=0.5$ than when $\gamma=1$ and $M_{y}$ attains extrema there, while at heights where $V=0, M_{y}$ varies slower when $\gamma=0.5$ than when $\gamma=1$ and $M_{x}$ attains extrema there. A noteworthy difference with respect to Figs. 5-6 is that in Figs. 10-11 both $U=0$ and $V=0$ are satisfied at two different heights within the displayed domain.

When $R i=5$ (Figs. 8-11(a,b)), there is very good agreement between WKB theory (solid lines) and the linear numerical model (circles), and both results differ little from those valid in the $R i \rightarrow \infty$ limit (dotted lines) (though slightly more than for the linear wind profile). When $R i=1$, however, Figs. 8-11(c,d) show that both components of the momentum flux increase at all levels, but especially near the surface, except for $M_{x}$ in Fig. 9(c), which instead decreases slightly (but increases in absolute value). The gradient of the momentum flux divergence therefore tends to increase globally. This is consistent with the behaviour of the surface drag, which is 

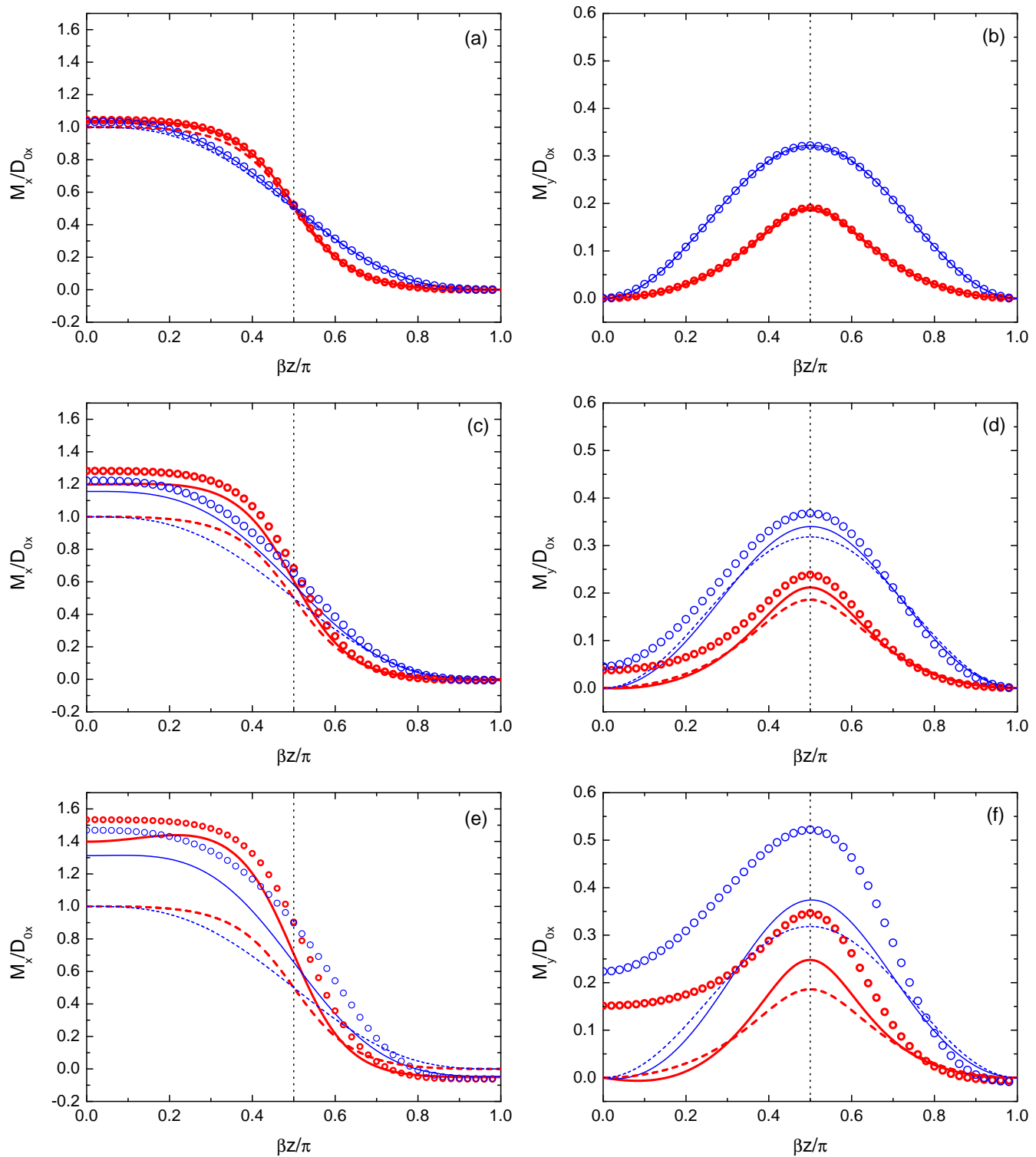

Figure 8: Similar to Fig. 3, but for the wind profile (48). The vertical dotted line denotes the height where $U=0(\beta z / \pi=1 / 2)$. 

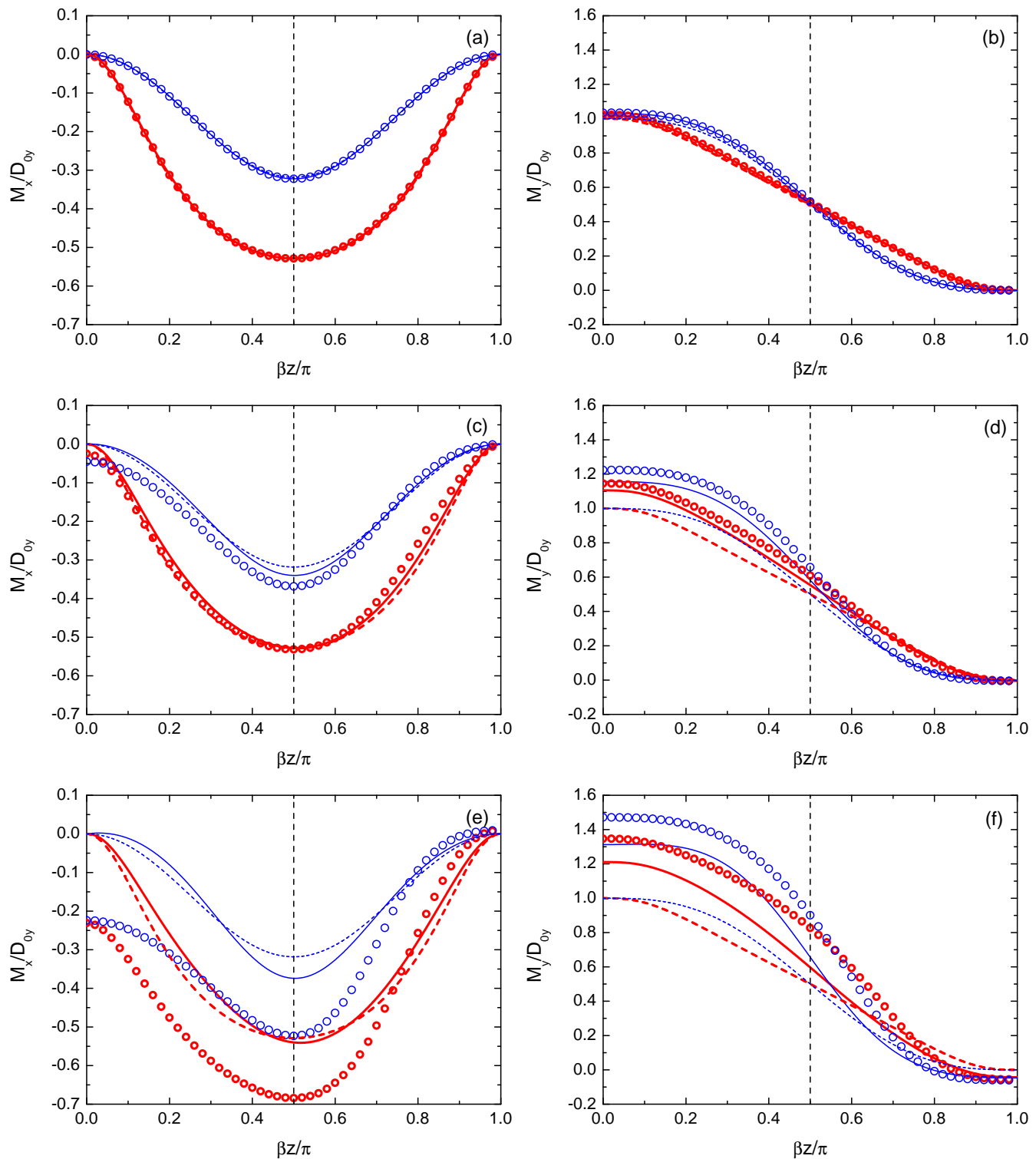

Figure 9: Similar to Fig. 4, but for the wind profile (48). The vertical dashed line denotes the height where $V=0(\beta z / \pi=1 / 2)$. 

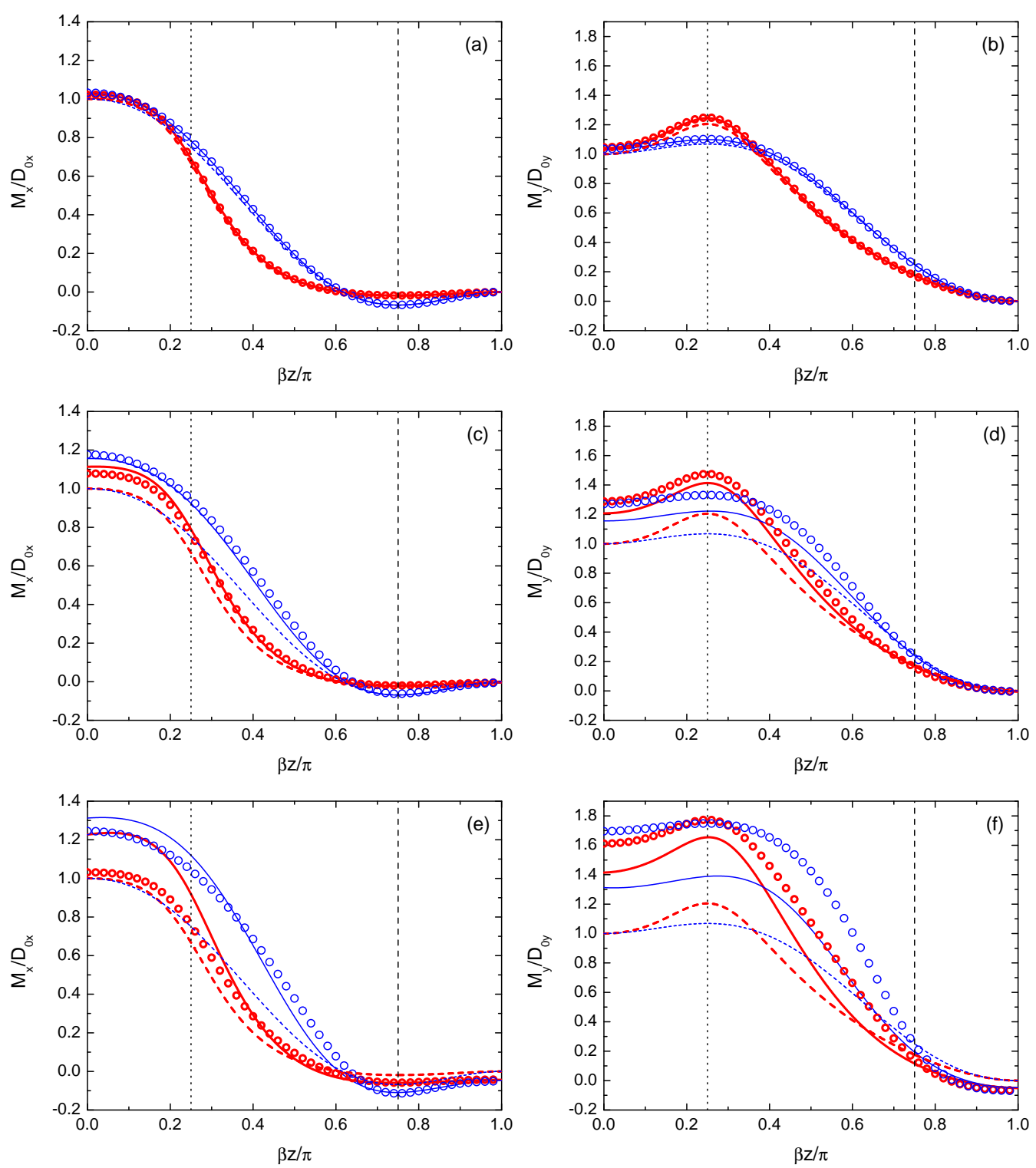

Figure 10: Similar to Fig. 5, but for the wind profile (48). The vertical dotted line denotes the height where $U=0(\beta z / \pi=1 / 4)$ and the vertical dashed line denotes the height where $V=0(\beta z / \pi=3 / 4)$. 

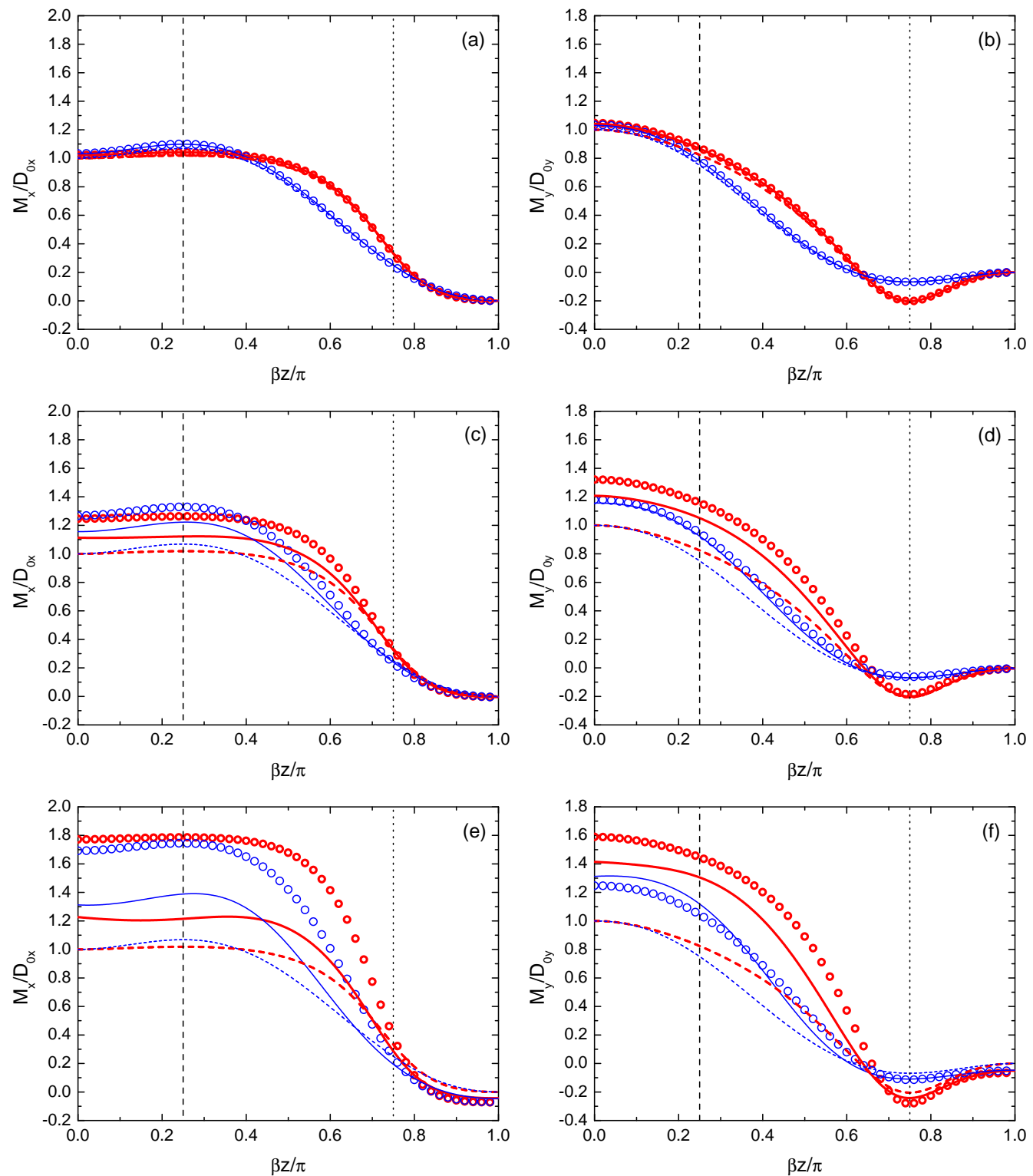

Figure 11: Similar to Fig. 6, but for the wind profile (48). The vertical dashed line denotes the height where $V=0(\beta z / \pi=1 / 4)$ and the vertical dotted line denotes the height where $U=0(\beta z / \pi=3 / 4)$. 
known to generally increase for this kind of wind profile $[14,16]$. The agreement between the WKB results and those from the linear numerical model is not as good as in Figs. 3-6. Generally, WKB theory underestimates the absolute value of the momentum flux compared with the linear numerical model, a notable exception being Fig. 10(c). Also worthy of note is the fact that WKB theory is unable to predict the non-zero value taken by the momentum flux at the surface (and near it) in the direction perpendicular to the flow at the surface. This is particularly striking in Figs. 8(d) and 9(c). Presumably, this limitation could only be overcome by extending the WKB approximation up to higher order. When $R i=0.5$ (Figs. 8-11(e,f)), the momentum flux is even more enhanced, with surface values in the direction of the incoming flow attaining normalized values of 1.6 or 1.8 (as given by the linear numerical model). For this value of $R i$, the agreement between these exact results and the approximate WKB results becomes less satisfactory, with a sizable underestimation of the absolute value of the momentum flux by WKB theory (except in Fig. 10(e) and for the thin blue line in Fig. 11(f), where there is overestimation instead). This underestimation is particularly severe for $M_{x}$ in Fig. 11(e). These discrepancies are related to both the tendency of the WKB approximation to underestimate the surface drag, and its inability to capture its rotation relative to the surface wind when $R i$ is relatively low, for this particular wind profile. Figs. 8(f) and 9(e) suggest that adding a function with a linear trend that fits the momentum flux at $z=0$ and $\beta z / \pi=1$ to $M_{y}$ and $M_{x}$, respectively, would greatly improve agreement between WKB theory and the linear numerical model, but this ad hoc procedure seems hardly justifiable. On the other hand, Figs. 8(e) and 
9(f) reveal that the underestimation, respectively, of $M_{x}$ and $M_{y}$ in those figures is not wholly due to nonlinear effects (as might be suggested by the numerical simulations of [17]), but is intrinsical to the WKB approximation.

Despite its imperfections, which undoubtedly are related with the presence of higher derivatives in this wind profile, and the truncation of the WKB approximation at third order, it is clear that WKB theory makes a much better job of predicting results from the linear numerical model than linear theory in the limit $R i \rightarrow \infty$. The gradients of $M_{x}$ in Fig. 8(e), of $M_{y}$ in Fig. 9(f), and of both $M_{x}$ and $M_{y}$ in Figs. 10(e,f) and 11(f) at mid-levels are predicted rather accurately by WKB theory, whereas linear theory with $R i \rightarrow \infty$ severely underestimates them. But agreement is not so satisfactory for $M_{y}$ in Fig. 8(f), $M_{x}$ in Fig. 9(e), or $M_{x}$ in Fig. 11(e).

\section{Concluding remarks}

Using a third-order WKB solution to the Taylor-Goldstein equation, linear theory has been employed to calculate the momentum flux associated with internal gravity waves in the atmosphere generated by steady, hydrostatic flow over mountains with an elliptical horizontal cross-section, for slowly-varying but otherwise generic wind profiles. This momentum flux decisively affects the intensity of westerly winds in mid-latitudes, modifying wave propagation in the atmosphere, and consequently also surface weather patterns. Via thermal-wind balance, upper-air temperatures in high latitudes [3], which control the formation of polar stratospheric clouds, are also affected, with implications for ozone depletion [25]. For all these reasons, this subgrid-scale physical process must be parametrized in large-scale weather 
and climate prediction models [26]. The present calculations aim to improve those parametrizations by incorporating a self-consistent treatment of vertical wind shear effects on the momentum flux.

The orographic drag parametrization currently included in the ECMWF weather prediction system [4] uses the concept of wave saturation associated with breaking of high-amplitude waves to determine the location and magnitude of the momentum deposition that corresponds to the reaction force exerted by mountains on the atmosphere, following an approach based on Lindzen [27]. This process depends on the vertical structure of the wind and static stability, but that is the only wind profile effect that enters into the parametrization. A description of how directional wind shear affects the surface drag [23], or the detailed momentum flux absorption process, is currently missing. However, even low-amplitude mountain waves are affected by directional wind shear, and their associated momentum flux varies in accordance with the extension to 3D of Eliassen-Palm's theorem [9]. At relatively low Richardson numbers, momentum deposition at critical levels may not be total, as assumed in the saturation hypothesis, or in the linear analyses of Shutts [6] and Shutts and Gadian [7], but rather partial, affecting the mean flow in distinct ways. Additionally, vertical wind shear also affects the surface drag, making it increase for certain types of wind profile and decrease for others $[14,16]$. The incorporation of these missing effects has long been overdue in drag parametrizations (which have become severely outdated), and they might help to alleviate known biases and imbalances in the global angular momentum budget [28].

The present study provides the necessary theory, in an essentially ready- 
to-use form, for implementing these changes. The momentum flux is calculated for mountains with an elliptical horizontal cross-section, which is the approximation used to represent the Earth's mountain ranges in each grid box in the ECMWF drag parametrization. The hydrostatic assumption (which is also used in the present calculations) then allows corrections to the momentum flux, or its divergence, to be cast in a form that is independent of the detailed shape of the orography, which makes the results much more general. The corrections to the momentum flux must be evaluated in a coordinate system aligned with the main axes of the mountain, which should be straightforward to do by applying the required coordinate transformation.

The results presented here show that the WKB approximation is accurate for calculating the momentum flux down to Richardson numbers as low as 0.5, leading to a substantial improvement relative to the infinite- $R i$ limit. Since the atmosphere becomes dynamically unstable at $R i=0.25$, this encompasses most of the relevant range of $R i$ likely to be realized in the atmosphere. While the linear assumption, used in all calculations, is restrictive, it seems to be unavoidable in the derivation of any physically consistent momentum flux expressions amenable to be implemented in drag parametrizations. Nonlinear effects, which have been studied extensively using 3D numerical simulations [29, 30], display a rich behaviour whose complexity is difficult to fully take into account. This explains perhaps, for example, the withdrawal of empirical nonlinear corrections initially included in the UK Meteorological Office's drag parametrization [31]. Clearly, a better understanding of these effects continues to be necessary.

A practical aspect that was addressed in the present paper was how to 
deal with the occurrence of non-monotonic wind profiles, or profiles where the wind turns by an angle larger than $\pi$, when each wavenumber present in the wave spectrum has more than one critical level. In both cases, the solution is to split the atmosphere in as many layers as necessary, so that within each layer each wavenumber has a single critical level. The momentum flux, or its divergence, is then expressed as a function of the momentum flux at the bottom of each layer, which is the same as the momentum flux at the top of the layer immediately below, calculated previously.

The present results extend the calculations of Shutts and Gadian [7] to lower Ri (as had been done by Teixeira and Miranda [17] for axisymmetric mountains), and extend the work of both of these authors to flow over elliptical mountains (as had been done for the surface drag by Teixeira and Miranda [16]). Therefore, these results, along with those of [17], complement the general constraint on the momentum flux derived by Broad [9] in a similar way as the studies of Booker and Bretherton [18] and Grubišić and Smolarkiewicz [19] (for much less general unidirectional wind profiles) complemented the Eliassen-Palm theorem [8].

\section{Appendix A. Definitions of $I_{1}-I_{6}$}

For a wind that turns monotonically anti-clockwise with height by an angle no larger than $\pi$, the integrals introduced in (13)-(14) are defined as follows:

$$
\begin{aligned}
& I_{1}=\int_{\psi-\pi / 2}^{\psi_{0}+\pi / 2} \frac{\cos ^{2} \theta}{\left(\cos ^{2} \theta+\gamma^{2} \sin ^{2} \theta\right)^{1 / 2}}[1-S(\theta, z)] e^{S(\theta, z)-S(\theta, z=0)} d \theta, \\
& I_{2}=\int_{\psi_{0}+\pi / 2}^{\psi+\pi / 2} \frac{\cos ^{2} \theta}{\left(\cos ^{2} \theta+\gamma^{2} \sin ^{2} \theta\right)^{1 / 2}}[1-S(\theta, z)] e^{S(\theta, z)-S(\theta, z=0)} e^{-2 \pi C(\theta)} d \theta,
\end{aligned}
$$




$$
\begin{aligned}
& I_{3}=\int_{\psi-\pi / 2}^{\psi_{0}+\pi / 2} \frac{\cos \theta \sin \theta}{\left(\cos ^{2} \theta+\gamma^{2} \sin ^{2} \theta\right)^{1 / 2}}[1-S(\theta, z)] e^{S(\theta, z)-S(\theta, z=0)} d \theta \\
& I_{4}=\int_{\psi_{0}+\pi / 2}^{\psi+\pi / 2} \frac{\cos \theta \sin \theta}{\left(\cos ^{2} \theta+\gamma^{2} \sin ^{2} \theta\right)^{1 / 2}}[1-S(\theta, z)] e^{S(\theta, z)-S(\theta, z=0)} e^{-2 \pi C(\theta)} d \theta \\
& I_{5}=\int_{\psi-\pi / 2}^{\psi_{0}+\pi / 2} \frac{\sin ^{2} \theta}{\left(\cos ^{2} \theta+\gamma^{2} \sin ^{2} \theta\right)^{1 / 2}}[1-S(\theta, z)] e^{S(\theta, z)-S(\theta, z=0)} d \theta \\
& I_{6}=\int_{\psi_{0}+\pi / 2}^{\psi+\pi / 2} \frac{\sin ^{2} \theta}{\left(\cos ^{2} \theta+\gamma^{2} \sin ^{2} \theta\right)^{1 / 2}}[1-S(\theta, z)] e^{S(\theta, z)-S(\theta, z=0)} e^{-2 \pi C(\theta)} d \theta .
\end{aligned}
$$

If the wind turns clockwise instead, with the other assumptions remaining the same, $\psi$ and $\psi_{0}$ should be exchanged in the above equations.

\section{Appendix B. Forms taken by $S(\theta, z), C(\theta), S_{\psi}(z)$ and $C_{\psi}(z)$}

For the two idealized wind profiles adopted in the present study, $S(\theta, z)$, $C(\theta), S_{\psi}(z)$ and $C_{\psi}(z)$, given in general by (8)-(9) and (33)-(34) simplify considerably.

\section{Linear wind profile}

For the linear wind profile (47), (8)-(9) reduce to

$$
\begin{aligned}
S(\theta, z)= & \frac{1}{8 R i} \frac{\left[\cos \theta \sin \left(\delta+\frac{\pi}{4}\right)-\gamma \sin \theta \cos \left(\delta+\frac{\pi}{4}\right)\right]^{2}}{\cos ^{2} \theta+\gamma^{2} \sin ^{2} \theta} \\
C(\theta)= & \frac{R i^{1 / 2}\left(\cos ^{2} \theta+\gamma^{2} \sin ^{2} \theta\right)^{1 / 2}}{\left|\cos \theta \sin \left(\delta+\frac{\pi}{4}\right)-\gamma \sin \theta \cos \left(\delta+\frac{\pi}{4}\right)\right|} \\
& \times\left\{1-\frac{1}{8 R i} \frac{\left[\cos \theta \sin \left(\delta+\frac{\pi}{4}\right)-\gamma \sin \theta \cos \left(\delta+\frac{\pi}{4}\right)\right]^{2}}{\cos ^{2} \theta+\gamma^{2} \sin ^{2} \theta}\right\}
\end{aligned}
$$


On the other hand, (33)-(34) reduce to

$$
\begin{aligned}
S_{\psi}(z)= & \frac{1}{8 R i} \frac{\left[\sin \psi \sin \left(\delta+\frac{\pi}{4}\right)+\gamma \cos \psi \cos \left(\delta+\frac{\pi}{4}\right)\right]^{2}}{\sin ^{2} \psi+\gamma^{2} \cos ^{2} \psi}, \\
C_{\psi}(z)= & \frac{R i^{1 / 2}\left(\sin ^{2} \psi+\gamma^{2} \cos ^{2} \psi\right)^{1 / 2}}{\left|\sin \psi \sin \left(\delta+\frac{\pi}{4}\right)+\gamma \cos \psi \cos \left(\delta+\frac{\pi}{4}\right)\right|} \\
& \times\left\{1-\frac{1}{8 R i} \frac{\left[\sin \psi \sin \left(\delta+\frac{\pi}{4}\right)+\gamma \cos \psi \cos \left(\delta+\frac{\pi}{4}\right)\right]^{2}}{\sin ^{2} \psi+\gamma^{2} \cos ^{2} \psi}\right\}
\end{aligned}
$$

where, from (10) and (47),

$$
\tan \psi=\gamma \frac{U_{0} \sin \delta+\alpha z \cos \left(\delta+\frac{\pi}{4}\right)}{U_{0} \cos \delta-\alpha z \sin \left(\delta+\frac{\pi}{4}\right)} .
$$

Using this equation, (B.3)-(B.4) may be further simplified as

$$
\begin{aligned}
S_{\psi}(z)= & \frac{1}{16 R i\left[1+\left(\frac{\alpha z}{U_{0}}\right)^{2}-\sqrt{2}\left(\frac{\alpha z}{U_{0}}\right)\right]}, \\
C_{\psi}(z)= & \sqrt{2} R i^{1 / 2}\left[1+\left(\frac{\alpha z}{U_{0}}\right)^{2}-\sqrt{2}\left(\frac{\alpha z}{U_{0}}\right)\right]^{1 / 2} \\
& \times\left\{1-\frac{1}{16 R i\left[1+\left(\frac{\alpha z}{U_{0}}\right)^{2}-\sqrt{2}\left(\frac{\alpha z}{U_{0}}\right)\right]}\right\} .
\end{aligned}
$$

Note that $S_{\psi}$ and $C_{\psi}$ do not depend on the aspect ratio of the mountain $\gamma$.

Wind that turns with height

For the wind that turns with height (48), (8)-(9) reduce to

$$
\begin{aligned}
S(\theta, z)= & \frac{1}{8 R i\left(\cos ^{2} \theta+\gamma^{2} \sin ^{2} \theta\right)}\left\{[\cos \theta \sin (\beta z+\delta)-\gamma \sin \theta \cos (\beta z+\delta)]^{2}\right. \\
& \left.-2[\cos \theta \cos (\beta z+\delta)+\gamma \sin \theta \sin (\beta z+\delta)]^{2}\right\} \\
C(\theta)= & R i^{1 / 2}\left(1-\frac{1}{8 R i}\right) .
\end{aligned}
$$


Note the very simple definition of $C(\theta)$, which in fact does not depend on $\theta$ and coincides with the one valid for an axisymmetric mountain in [17]. On the other hand, (33)-(34) reduce to

$$
\begin{aligned}
S_{\psi}(z)= & \frac{1}{8 R i\left(\sin ^{2} \psi+\gamma^{2} \cos ^{2} \psi\right)}\left\{[\sin \psi \sin (\beta z+\delta)+\gamma \cos \psi \cos (\beta z+\delta)]^{2}\right. \\
& \left.-2[\sin \psi \cos (\beta z+\delta)-\gamma \cos \psi \sin (\beta z+\delta)]^{2}\right\}, \\
C_{\psi}(z)= & R i^{1 / 2}\left(1-\frac{1}{8 R i}\right),
\end{aligned}
$$

where

$$
\tan \psi=\gamma \tan (\beta z+\delta)
$$

If this equation is used, it can be shown that (B.10) simplifies further to

$$
S_{\psi}(z)=\frac{1}{8 R i}
$$

For this wind profile, $S_{\psi}$ and $C_{\psi}$ are also independent of $\gamma$, and in fact independent of height, being equal to those valid for an axisymmetric mountain in $[17]$.

\section{Acknowledgements}

The authors are grateful to Tor Sivertsen and two anonymous referees for useful comments, which have improved the revised version of this paper. M.A.C.T. acknowledges the financial support of the Portuguese Science Foundation (FCT) under Grant PTDC/CTE-ATM/122501/2010 and of the European Union, under Marie Curie Career Integration Grant GLIMFLO, contract PCIG13-GA-2013-618016. 


\section{References}

[1] C. J. Nappo, An Introduction to Atmospheric Gravity Waves - Second Edition, Academic Press, 2012.

[2] Y.-L. Lin, Mesoscale Dynamics, Cambridge University Press, 2007.

[3] T. N. Palmer, G. J. Shutts, R. Swinbank, Alleviation of a systematic westerly bias in general circulation and numerical weather prediction models through an orographic gravity wave drag parametrization, Quart. J. Roy. Meteor. Soc. 112 (1986) 1001-1039.

[4] F. Lott, M. J. Miller, A new subgrid-scale orographic drag parametrization: its formulation and testing, Quart. J. Roy. Meteor. Soc. 123 (1997) $101-127$.

[5] D. Gregory, G. J. Shutts, J. R. Mitchell, A new gravity-wave-drag scheme incorporating anisotropic orography and low-level wave breaking. impact upon the climate of the uk meteorological office unified model, Quart. J. Roy. Meteor. Soc. 124 (1998) 463-493.

[6] G. Shutts, Gravity-wave drag parametrization over complex terrain: The effect of critical-level absorption in directional wind-shear, Quart. J. Roy. Meteor. Soc. 121 (1995) 1005-1021.

[7] G. J. Shutts, A. Gadian, Numerical simulations of orographic gravity waves in flows which back with height, Quart. J. Roy. Meteor. Soc. 125 (1999) 2743-2765. 
[8] A. Eliassen, E. Palm, On the transfer of energy in stationary mountain waves, Geofys. Publ. 22 (1961) 1-23.

[9] A. S. Broad, Linear theory of momentum fluxes in 3-d flows with turning of the mean wind with height, Quart. J. Roy. Meteor. Soc. 121 (1995) 1891-1902.

[10] M. A. C. Teixeira, P. M. A. Miranda, J. L. Argaín, M. A. Valente, Resonant gravity-wave drag enhancement in linear stratified flow over mountains, Quart. J. Roy. Meteor. Soc. 131 (2005) 1795-1814.

[11] M. A. C. Teixeira, P. M. A. Miranda, J. L. Argaín, Mountain waves in two-layer sheared flows: critical level effects, wave reflection and drag enhancement, J. Atmos. Sci. 65 (2008) 1912-1926.

[12] M. A. C. Teixeira, J. L. Argaín, P. M. A. Miranda, Drag produced by trapped lee waves and propagating mountain and propagating mountain waves in a two-layer atmosphere, Quart. J. Roy. Meteor. Soc. 139 (2013) 964-981.

[13] M. A. C. Teixeira, J. L. Argaín, P. M. A. Miranda, Orographic drag associated with lee waves trapped at an inversion, J. Atmos. Sci. 70 (2013) 2930-2947.

[14] M. A. C. Teixeira, P. M. A. Miranda, M. A. Valente, An analytical model of mountain wave drag for wind profiles with shear and curvature, J. Atmos. Sci. 61 (2004) 1040-1054.

[15] M. A. C. Teixeira, P. M. A. Miranda, The effect of wind shear and 
curvature on the gravity wave drag produced by a ridge, J. Atmos. Sci. 61 (2004) 2638-2643.

[16] M. A. C. Teixeira, P. M. A. Miranda, A linear model of gravity wave drag for hydrostatic sheared flow over elliptical mountains, Quart. J. Roy. Meteor. Soc. 132 (2006) 2439-2458.

[17] M. A. C. Teixeira, P. M. A. Miranda, On the momentum fluxes associated with mountain waves in directionally sheared flows, J. Atmos. Sci. 66 (2009) 3419-3433.

[18] J. R. Booker, F. P. Bretherton, The critical layer for internal gravity waves in a shear flow, J. Fluid Mech. 27 (1967) 513-539.

[19] V. Grubišić, P. K. Smolarkiewicz, The effect of critical levels on 3d orographic flows: linear regime, J. Atmos. Sci. 54 (1997) 1943-1960.

[20] P. G. Baines, T. N. Palmer, Rationale for a new physically based parametrization of sub-grid scale orographic effects, Tech. Memo. 169, European Centre for Medium-Range Weather Forecasts (1990).

[21] M. A. C. Teixeira, P. M. A. Miranda, R. M. Cardoso, Asymptotic gravity wave drag expressions for non-hydrostatic rotating flow over a ridge, Quart. J. Roy. Meteor. Soc. 134 (2008) 271-276.

[22] P. M. A. Miranda, J. P. A. Martins, M. A. C. Teixeira, Assessing wind profile effects on the global atmospheric torque, Quart. J. Roy. Meteor. Soc. 135 (2009) 807-814. 
[23] D. S. Phillips, Analytical surface pressure and drag for linear hydrostatic flow over three-dimensional elliptical mountains, J. Atmos. Sci. 41 (1984) 1073-1084.

[24] T. H. Sivertsen, Om stasjonære fjellbølger, Master's thesis, University of Oslo, Norway (1972).

[25] S. Solomon, Stratospheric ozone depletion: a review of concepts and history, Rev. Geophys. 37 (1999) 275-316.

[26] D. J. Stensrud, Parametrization Schemes: Keys to Understanding Numerical Weather Prediction Models, Cambridge University Press, 2009.

[27] R. S. Lindzen, Turbulence and stress owing to gravity wave and tidal breakdown, J. Geophys. Res. 86 (1981) 9707-9714.

[28] J. Egger, K. P. Hoinka, The annual cycle of the axial angular momentum of the atmosphere, J. Climate 18 (2005) 757-771.

[29] P. M. A. Miranda, I. N. James, Non-linear three dimensional effects on the wave drag: splitting flow and breaking waves, Quart. J. Roy. Meteor. Soc. 118 (1992) 1057-1081.

[30] H. Ólafsson, P. Bougeault, Nonlinear flow past an elliptic mountain ridge, J. Atmos. Sci. 53 (1996) 2465-2489.

[31] S. Webster, A. R. Brown, D. R. Cameron, C. P. Jones, Improvements to the representation of orography in the met office unified model, Quart. J. Roy. Meteor. Soc. 129 (2003) 1989-2010. 